\title{
Hex1, the Major Component of Woronin Bodies, Is Required for Normal Development, Pathogenicity, and Stress Response in the Plant Pathogenic Fungus Verticillium dahliae
}

\author{
Vasileios Vangalis $\left.{ }^{1}{ }^{(}\right)$, Ioannis A. Papaioannou ${ }^{2}{ }^{(D)}$, Emmanouil A. Markakis ${ }^{3}{ }^{(D)}$, \\ Michael Knop ${ }^{2,4}$ and Milton A. Typas ${ }^{1, *}$ \\ 1 Department of Genetics \& Biotechnology, Faculty of Biology, National and Kapodistrian University of \\ Athens, 15784 Athens, Greece; vasvagg@biol.uoa.gr \\ 2 Center for Molecular Biology, Heidelberg University (ZMBH), 69120 Heidelberg, Germany; \\ i.papaioannou@zmbh.uni-heidelberg.de (I.A.P.); m.knop@zmbh.uni-heidelberg.de (M.K.) \\ 3 Laboratory of Mycology, Department of Viticulture, Vegetable Crops, Floriculture and Plant Protection, \\ Institute of Olive Tree, Subtropical Crops and Viticulture, N.A.G.R.E.F., Hellenic Agricultural \\ Organization_DEMETER, 71307 Heraklion, Crete, Greece; markakis@nagref-her.gr \\ 4 German Cancer Research Center (DKFZ), DKFZ-ZMBH Alliance, 69120 Heidelberg, Germany \\ * Correspondence: matypas@biol.uoa.gr
}

Received: 6 November 2020; Accepted: 3 December 2020; Published: 7 December 2020

\begin{abstract}
Woronin bodies are membrane-bound organelles of filamentous ascomycetes that mediate hyphal compartmentalization by plugging septal pores upon hyphal damage. Their major component is the peroxisomal protein Hex1, which has also been implicated in additional cellular processes in fungi. Here, we analyzed the Hex1 homolog of Verticillium dahliae, an important asexual plant pathogen, and we report its pleiotropic involvement in fungal growth, physiology, stress response, and pathogenicity. Alternative splicing of the Vdhex 1 gene can lead to the production of two Hex1 isoforms, which are structurally similar to their Neurospora crassa homolog. We show that $V d \mathrm{Hex} 1$ is targeted to the septum, consistently with its demonstrated function in sealing hyphal compartments to prevent excessive cytoplasmic bleeding upon injury. Furthermore, our investigation provides direct evidence for significant contributions of Hex 1 in growth and morphogenesis, as well as in asexual reproduction capacity. We discovered that Hex1 is required both for normal responses to osmotic stress and factors that affect the cell wall and plasma-membrane integrity, and for normal resistance to oxidative stress and reactive oxygen species (ROS) homeostasis. The Vdhex 1 mutant exhibited diminished ability to colonize and cause disease on eggplant. Overall, we show that Hex1 has fundamentally important multifaceted roles in the biology of $V$. dahliae.
\end{abstract}

Keywords: septal pore; hyphal integrity; virulence; ROS; heterokaryon incompatibility

\section{Introduction}

The undifferentiated body (or thallus) of a typical filamentous fungus is the mycelium, a complex network of branched tubular cells called hyphae. In most fungi, these are partitioned into distinct cellular compartments by internal cross-walls, the septae [1,2]. Hyphal compartments are continuous with one another due to the occurrence of septal pores, which allow the intercellular flow of cytoplasm, including organelles, ensuring the rapid translocation of nutrients during colony establishment and facilitating the maintenance of cellular homeostasis during mycelial growth [3]. However, this ability of the mycelium to function as an integrated syncytium-like organism exposes it to a number of risks, 
e.g., excessive cytoplasmic leakage upon hyphal wounding or uncontrolled spread of selfish genetic elements (such as mycoviruses, transposable elements, and senescence plasmids) following vegetative hyphal fusion. Therefore, the intercompartmental traffic in hyphae must be highly regulated in order to protect the mycelium from such hazards, as well as to permit cellular heterogeneity and differentiation during developmental processes [3-7].

Early studies in various ascomycetes implicated a specialized membrane-bound vesicle described as Woronin body (WB) in sealing of septal pores to prevent cytoplasmic loss in response to hyphal damage $[8,9]$. It was later shown that WBs are involved in the control of intercellular communication and the maintenance of hyphal heterogeneity [10]. Woronin bodies, which are exclusively found in Pezizomycotina [9], originate from peroxisomes, and their biogenesis requires multiple peroxins [11]. They appear in electron microscopy images as electron-dense vesicles that are usually localized in close proximity to the septae, and they are rapidly tethered to the septal pore by interacting proteins following hyphal wounding [12,13]. At least 17 septal pore-associated WB-related proteins have been detected by mass spectrometry in Neurospora crassa [14].

The protein Hex1 was first characterized in N. crassa as the major component of WBs [15]. This protein spontaneously self-assembles into hexagonal crystals that comprise the dense core of WBs [16]. Conserved homologs of the hex 1 gene have been identified and studied in several members of the Pezizomycotina [17-26]. The gene presumably resulted from duplication of the ancestral gene encoding the eukaryotic initiation factor 5A (eIF-5A) [16]. After this duplication, hex1 followed a different evolutionary trajectory that led to a new function by acquiring those amino acids that are responsible for its peroxisomal targeting and self-assembly. Hex 1 genes retain a conserved intron close to their $\mathrm{N}$-terminus, and alternative splicing of this intron has been shown to produce two isoforms of the protein [17-19].

Deletion of hex1 in several fungi generally led to excessive hyphal bleeding after wounding [20,27], as well as had pleiotropic effects on additional phenotypes associated with vegetative growth, asexual reproduction, and stress response against osmotic and cell wall-perturbating agents [24-26]. Regarding pathogenicity and virulence, conflicting results have been gathered from studies on plant, insect, and human pathogens. These range from important roles of Hex1 in the formation of appressoria (infection structures) and pathogenicity in Magnaporthe grisea [18] to moderately reduced or delayed virulence in Fusarium graminearum [22], Aspergillus flavus [25], and A. fumigates [13], and no significant defects in pathogenicity of Colletotrichum orbiculare [23] and Metarhizium robertsii [26].

The ascomycete Verticillium dahliae causes a wilt disease on a wide range of commercially important plants and crops, and it is responsible for enormous annual economic losses worldwide [28]. Its resting structures (microsclerotia), which can remain viable in the soil for several years, germinate upon induction by exudates from adjacent plant roots to form hyphae. This is followed by root penetration and colonization, leading the fungus to the host vascular system, through which it can cause systemic infection of the plant [29]. Except for the apical cells of its mycelium, hyphal compartments of $V$. dahliae are regularly septated, and, upon hyphal injury, WBs rapidly plug the pores of the flanking septae to seal damaged compartments [30,31]. This fungus is thought to completely lack a sexual stage and, thus, propagates exclusively through the dispersion of its asexual spores (conidia). A parasexual cycle, initiated by hyphal fusion between individuals with different genotypes to the formation of heterokaryons, has been described in Verticillium and is thought to increase genetic diversity through random chromosome assortment and frequent mitotic recombination [28,32]. Nevertheless, heterokaryon formation is restricted by incompatibility barriers [28,31], which seal and destroy incompatible fused cells, while the remaining mycelium remains unaffected $[7,33]$.

This study aimed at the identification and functional characterization of hex 1 in fundamental biological processes of the important plant pathogen $V$. dahliae. For this, we deleted its hex 1 homolog to address the involvement of the gene in hyphal integrity, growth and development, pathogenicity, response to osmotic, cell wall-perturbating, and oxidative stress, and heterokaryon incompatibility. Our investigations revealed important roles of Hex1 in the biology of V. dahliae. 


\section{Materials and Methods}

\subsection{Fungal Strains, Culture Conditions, and Fungal DNA Isolation and Manipulation}

All fungal strains that were constructed and used in this study are listed in Table S1 (Supplementary Materials). Preparation and maintenance of monoconidial strains, culture media, and conditions were previously described [34].

Total genomic DNA of fungal strains was extracted according to published methods [34]. Standard and previously described procedures were followed for PCR amplification (all oligos are listed in Table S2, Supplementary Materials), cloning and maintenance of plasmids in E. coli strain $\mathrm{DH} 5 \alpha$ (a list of plasmids is provided in Table S3, Supplementary Materials), restriction digestion, and Sanger sequencing [34,35].

\subsection{Agrobacterium tumefaciens-Mediated Transformation of V. dahliae}

The hypervirulent $A$. tumefaciens strain AGL-1 was transformed with binary plasmid vectors using a $\mathrm{CaCl}_{2}$ /heat shock-mediated freeze-thaw method [36]. The resulting $A$. tumefaciens strains were used for transforming $V$. dahliae with protocols modified from [37]. After growth of $A$. tumefaciens in minimal medium (MM) supplemented with the proper selective antibiotics for $48 \mathrm{~h}\left(28^{\circ} \mathrm{C}, 180 \mathrm{rpm}\right)$, the culture was diluted to $\mathrm{OD}_{600}=0.1$ and incubated for $6 \mathrm{~h}$ in acetosyringone-containing induction medium (IM; $25^{\circ} \mathrm{C}, 180 \mathrm{rpm}$, without antibiotics). Aliquots of this culture (100 $\left.\mu \mathrm{L}\right)$ were then mixed with $100 \mu \mathrm{L}$ aliquots of a conidial suspension of the desired $V$. dahliae strain $\left(10^{7}\right.$ conidia/mL, prepared from 7 day old cultures) and plated on sterile cellophane sheets on IM agar. The mixture was incubated for $48 \mathrm{~h}$ at $25^{\circ} \mathrm{C}$ before its transfer to selective medium (potato dextrose agar, PDA) amended with $200 \mu \mathrm{g} / \mathrm{mL}$ cefotaxime, $50 \mu \mathrm{g} / \mathrm{mL}$ 5-fluorouracil, and $100 \mu \mathrm{g} / \mathrm{mL}$ geneticin and/or $15 \mu \mathrm{g} / \mathrm{mL}$ hygromycin B).

\subsection{Protoplast Transformation of V. dahliae}

At least $10^{8}$ conidia of the desired $V$. dahliae strain were incubated in Czapek-Dox complete medium (CzD-CM) for $16 \mathrm{~h}\left(25^{\circ} \mathrm{C}, 175 \mathrm{rpm}\right)$. Conidia/germlings were then pelleted, resuspended into a phosphate- $\mathrm{KCl}$ buffer $\left(0.6 \mathrm{M} \mathrm{KCl}, 1 / 15 \mathrm{M} \mathrm{KH}_{2} \mathrm{PO}_{4}\right.$, and 1/15 $\mathrm{M} \mathrm{Na}_{2} \mathrm{HPO}_{4}, \mathrm{pH}$ 5.8; supplemented with $25 \mathrm{mM}$ dithiothreitol (DTT) and incubated for $2 \mathrm{~h}$ at $30^{\circ} \mathrm{C}(50 \mathrm{rpm})$. Protoplastation was performed by transferring the treated germlings to fresh phosphate- $\mathrm{KCl}$ buffer supplemented with $8 \mathrm{mg} / \mathrm{mL}$ of a lysing enzyme mix from Trichoderma harzianum (Sigma-Aldrich, St. Louis, MO, USA) and further incubating the mix at $30^{\circ} \mathrm{C}(50 \mathrm{rpm})$ for $1-3 \mathrm{~h}$. Protoplasts were separated from aggregated cellular debris by filtering through four layers of sterile gauge and resuspended into STC solution (1.2 M sorbitol, $10 \mathrm{mM}$ Tris-Cl pH 7.5, and $\left.50 \mathrm{mM} \mathrm{CaCl}_{2}\right)$ at a final concentration of $10^{8} / \mathrm{mL}$. Aliquots $(100 \mu \mathrm{L})$ of this protoplast suspension were mixed with 2-8 $\mu \mathrm{g}$ of DNA and incubated for 30 min on ice before the addition of $200 \mu \mathrm{L}$ of PTC buffer (40\% PEG-6000, $25 \mathrm{mM}$ Tris-Cl pH 7.5 and $25 \mathrm{mM} \mathrm{CaCl}_{2}$ ) and further incubation for $20 \mathrm{~min}$ on ice. This was followed by the stepwise addition of $1 \mathrm{~mL}$ of fresh PTC buffer and incubation for $15 \mathrm{~min}$ on ice. The mixture was then washed three times with STC buffer, resuspended into fresh STC buffer, and plated on regeneration agar medium (20.54 g sucrose, $0.1 \mathrm{~g}$ yeast extract, and $0.14 \mathrm{~g}$ casamino acids per $100 \mathrm{~mL}$ ), overlaid with a sterile cellophane sheet. Following incubation at $25^{\circ} \mathrm{C}$ for $24 \mathrm{~h}$, the cellophane sheet was transferred onto the appropriate selective medium (PDA amended with $100 \mu \mathrm{g} / \mathrm{mL}$ geneticin and/or $15 \mu \mathrm{g} / \mathrm{mL}$ hygromycin B).

\subsection{Deletion, Complementation, and sGFP-Tagging of V. dahliae hex1}

The NEBuilder HiFi DNA Assembly Master Mix (New England Biolabs, Ipswich, MA, USA) was used (according to the manufacturer's recommendations) for the construction, in two steps, of a plasmid vector for hex 1 deletion by homologous recombination. The first reaction resulted in a plasmid containing 2000 bp long homologous arms (amplified from the genomic DNA of $V$. dahliae isolate Ls.17 using the Herculase II Fusion DNA polymerase; Agilent, Santa Clara, CA, USA) flanking the neo cassette (conferring resistance to geneticin) from plasmid pSD1, in the backbone of plasmid 
pOSCAR. A second NEBuilder HiFi DNA Assembly reaction was then used for the addition to the construct of the $h s v-t k$ thymidine kinase gene (from plasmid pGKO2) for selection against ectopic transformants. This recombinant plasmid vector (pOSCAR-hex-KO) was used for transformation of $V$. dahliae, using the Agrobacterium tumefaciens strain AGL-1. Monoconidial cultures were prepared from resistant $V$. dahliae colonies, validated as hex 1 deletion mutants by spore PCR and Southern blot analysis (DIG DNA Labeling and Detection Kit, Sigma-Aldrich), and stored as 25\% glycerol stocks at $-80^{\circ} \mathrm{C}$.

The full-length coding sequence of hex 1 plus $2000 \mathrm{bp}$ long flanks were amplified from the genomic DNA of $V$. dahliae Ls.17 (using the KAPA HiFi DNA polymerase, Roche, Basel, Switzerland) for complementing hex 1 deletion strains. The resulting $4.8 \mathrm{~kb}$ long amplicon was co-transformed into $V$. dahliae protoplasts with plasmid pUCATPH, which contains the $h p h$ cassette (conferring resistance to hygromycin B). Monoconidial transformants were confirmed by spore PCR and stored at $-80^{\circ} \mathrm{C}$.

We performed C-terminal sGFP-tagging of hex1 by using the NEBuilder HiFi DNA Assembly kit to generate a construct consisting of the full-length hex1 open reading frame (ORF) together with its $2000 \mathrm{bp}$ long upstream flank (which presumably includes its endogenous promoter) fused to the $s G F P$ coding sequence from plasmid pIGPAPA, the terminal region of A. nidulans tef1, and the $h p h$ selection cassette from plasmid pFC332. The Herculase II Fusion DNA Polymerase was used for the amplification of all fragments, which were then assembled into a new recombinant plasmid (pOSCAR-hex-GFP) in the backbone of pOSCAR by a NEBuilder reaction. This was then transformed into $V$. dahliae $\Delta$ hex 1 mutants using the $A$. tumefaciens strain AGL-1. Resistant fungal colonies were single-cell purified, microscopically checked, and stored at $-80^{\circ} \mathrm{C}$.

\subsection{Cytoplasmic and Nuclear Fluorescent Labeling of V. dahliae}

The SGFP gene was used for fluorescent labeling of either the cytoplasm or histone $\mathrm{H} 1$ (as a nuclear label) of $V$. dahliae strains. Construction of strains with cytoplasmic sGFP expression was performed by cloning the $S G F P$ expression cassette from plasmid pIGPAPA and the neo cassette from plasmid pSD1 to the polylinker of plasmid pBluescript II (using the T4 DNA ligase, Takara Bio, Kusatsu, Japan). The resulting plasmid pBS-GFP-gen was used to transform $V$. dahliae protoplasts, and monoconidial transformants were checked microscopically for robust cytoplasmic sGFP expression.

Plasmid pMF357, which carries the hph cassette and a fusion construct of the sGFP gene to the $N$. crassa histone $\mathrm{H} 1$ gene, was used to transform $V$. dahliae protoplasts for nuclear labeling. Monoconidial transformants were checked microscopically for green nuclear fluorescence.

\subsection{Morphological and Physiological Characterization of V. dahliae Strains}

Previously described methods [34] were used for the morphological and physiological characterization of wild-type and mutant $V$. dahliae strains, with minor modifications. Colony diameters were measured for the determination of growth rates every 5 days during growth on CzD-CM, at $25^{\circ} \mathrm{C}$. Mycelial plugs ( $1.0 \mathrm{~cm}$ in diameter) from the colony periphery of 20 day old cultures were transferred into $500 \mu \mathrm{L}$ of sterile water and vortexed extensively before determining conidial concentrations using a Neubauer improved cell counting chamber. Frequency of germination was determined by inoculating $1.0 \times 10^{6}$ conidia of each strain into CM and checking microscopically for germling formation after $14 \mathrm{~h}$ of growth at $25^{\circ} \mathrm{C}$. All experiments were performed in triplicate.

\subsection{Plant Pathogenicity Assays}

Preparation of eggplant seedlings and conidial suspensions from $V$. dahliae cultures for their pathogenicity assessment was performed as previously described [38], with minor modifications. Seedlings were transplanted at the one true-leaf stage in $100 \mathrm{~mL}$ pots containing soil substrate, before drenching with $20 \mathrm{~mL}$ of conidial suspension $\left(5.0 \times 10^{6}\right.$ conidia $\left./ \mathrm{mL}\right)$ or sterile water (control plants) per pot. Plants were maintained under controlled conditions at $23 \pm 3{ }^{\circ} \mathrm{C}$ with a $12 \mathrm{~h}$ light/dark cycle. 
Typical wilt symptoms were recorded every 3-4 days for 29 days post inoculation (d.p.i.) for monitoring disease progress over time, and at 45 d.p.i. for the determination of final disease severity. Disease parameters were recorded according to previously described criteria [38,39]. Disease severity at each time point was defined as the percentage of symptomatic leaves, and results were plotted over time to generate disease progress curves. These were used for the determination of relative AUDPC (area under disease progress curve) values [38]. In addition, plant growth parameters were recorded at 29 d.p.i. [39].

The presence of the applied $V$. dahliae strain in plant tissues was assessed by fungal re-isolation to verify systemic infection at 29 d.p.i., according to [38]. In particular, nine plant stems per treatment were randomly selected and three xylem chips from different sites along the stem (base, middle, and upper part) were transferred onto acidified PDA medium after the removal of the phloem. The pathogen isolation ratio was expressed as the percentage of positive xylem chips of each plant.

\subsection{Stress Response Assays and Reactive Oxygen Species (ROS) Detection}

Two methods were used to analyze the sensitivity of $V$. dahliae wild-type and mutant strains to varying concentrations of a number of stress-inducing factors and chemicals that are commercially used as fungicides. In summary, we tested growth responses to factors that induce osmotic stress $(\mathrm{NaCl}$, sorbitol), oxidative stress $\left(\mathrm{H}_{2} \mathrm{O}_{2}\right.$, paraquat, $\mathrm{N}$-acetyl cysteine, iprodione), cell wall/plasma-membrane damage or perturbation (calcofluor white M2R, Congo red, SDS, amphotericin B (Biosera, Nuaille, France), fluconazole (Pfizer, Brooklyn, NY, USA), farnesol), and other fungicides that inhibit sporulation and germination (cymoxanil, fosetyl-Al (Bayer, Leverkusen, Germany), mandipropamid, cyflufenamid); chemicals were purchased from Sigma-Aldrich, unless otherwise specified, and fresh solutions were prepared in water directly before each experiment. The first method was a spot dilution assay, in which $10 \mu \mathrm{L}$ of 10 -fold serial dilutions of conidial suspensions $\left(5.0 \times 10^{6}\right.$ to $5.0 \times 10^{3}$ conidia/mL) were spotted on $\mathrm{CM}$ agar plates containing the desired stress factor and incubated at $25^{\circ} \mathrm{C}$ for 3 days. In addition, for each stress condition/concentration, relative growth inhibition was determined according to the formula: \% growth inhibition $=(($ colony diameter on $\mathrm{CM}-$ colony diameter in stress condition $) /($ colony diameter on $\mathrm{CM})) \times 100$. For each substance considered, a range of concentrations were tested in preliminary experiments for the determination of the minimum inhibitory concentration (MIC) for the wild-type strain, and an appropriate value above the MIC yielding reproducible results between replicates was chosen for further analyses. Each experiment was performed in triplicate.

The generation/accumulation of superoxide anion radicals $\left(\mathrm{O}_{2}{ }^{-}\right)$in the mycelium of $V$. dahliae strains was also examined in response to stress-inducing compounds. For this, the nitro blue tetrazolium chloride (NBT; Cayman Chemical, Ann Arbor, MI, USA) staining method was used; fungal colonies were grown on $\mathrm{CM}$ plates supplemented with the desired stress factor for 20 days before the addition of $10 \mathrm{~mL}$ of a $0.2 \%$ NBT solution on each plate. Following incubation at $25^{\circ} \mathrm{C}$ (in the dark) for $45 \mathrm{~min}$, the solution was discarded; then, plates were washed with ethanol, incubated again for $45 \mathrm{~min}$ in the dark, and briefly air-dried before recording the assessment of the staining result. All staining assays were performed in triplicate.

\subsection{Generation of Nit Mutants and Heterokaryon Compatibility Tests}

Nitrate non-utilizing (nit) mutants of the $V$. dahliae $\Delta$ hex 1 strain were generated by the ultraviolet irradiation method and selection on the water agar chloratemedium (WAC), as previously described [40]. Phenotypic classification of nit mutants and complementation tests in 96-well plates were also performed according to our previously described procedures [40]. Each pairing was performed in three independent repetitions.

\subsection{Microscopy}

Fungal conidia, germlings, and hyphae were imaged using a Zeiss Axioplan epifluorescence microscope equipped with a differential interference contrast (DIC) optical system, a set of filters G 365 
$\mathrm{nm}$ (excitation) and LP $420 \mathrm{~nm}$ (emission), and a Zeiss Axiocam MRc5 digital camera. Calcofluor white M2R (Sigma-Aldrich) was used for cell wall staining by its addition to the samples (to a final concentration of $10 \mu \mathrm{g} / \mathrm{mL}$ ) and incubation at room temperature for $5 \mathrm{~min}$ before imaging. Methylene blue staining (final concentration $0.005 \% w / v$ ) was used to differentiate live from dead cells. The samples were incubated at $25^{\circ} \mathrm{C}$ for $5 \mathrm{~min}$, in the dark, before imaging.

Time-lapse microscopy was performed using a Nikon Ti-E epifluorescence microscope equipped with an autofocus system (Perfect Focus System, Nikon, Minato, Japan), a light-emitting diode (LED) light engine (SpectraX, Lumencor, Beaverton, OR, USA), filter sets 390/18 and 435/48 or 469/35 and 525/50 (excitation and emission filter, respectively; all from Semrock, Rochester, NY, USA except for 525/50, which was from Chroma, Bellows Falls, VT, USA), and a scientific complementary metal-oxide-semiconductor (sCMOS) camera (Flash4, Hamamatsu, Shizuoka, Japan). Images were acquired every $10 \mathrm{~min}$ for $24 \mathrm{~h}$ (with exposure of 50 and $20 \mathrm{~ms}$ for the green and blue channel, respectively), and later processed using Image [41]. Images were adjusted to uniform contrast across all time points, pixel intensity plots were generated using the plot profile (for septae) and surface plot (for the whole area) functions of ImageJ, and background subtraction was performed with a rolling ball radius of 50.0 pixels and default parameters.

\subsection{Bioinformatic Analyses and Statistics}

Homology searches for the identification of Hex1 orthologs in $V$. dahliae and other fungal species were performed using the basic local alignment search tools BlastN, tBlastN, and BlastP searches (NCBI; https://blast.ncbi.nlm.nih.gov/Blast.cgi). All protein sequences were retrieved from GenBank (NCBI; https://www.ncbi.nlm.nih.gov/genbank) and aligned using the MUSCLE algorithm implemented in MEGA X [42]; the alignment was then improved using ClustalW and manually corrected. Reads from previously published RNA-seq experiments of $V$. dahliae were obtained from the Sequence Read Archive (NCBI SRA; https://www.ncbi.nlm.nih.gov/sra; datasets with accession numbers SRP020910, SRP013922, SRP041118, SRP119401, SRP198907, SRP183605, SRP244752, ERP123035, and ERP002524) using BLAST analyses and aligned to the hex1 coding sequences that flank the predicted intron, using MEGA $X$.

The maximum likelihood (ML) method, implemented in the MEGA X software suite, was used for the generation of the phylogenetic tree of Hex1 sequences. Sequence alignment was performed using the MUSCLE algorithm, and then it was manually corrected. The Le Gascuel (LG) model, using a discrete Gamma distribution (parameter $=0.4452$ ) with five rate categories, exhibited the lowest Bayesian information criterion (BIC) and corrected Akaike information criterion (AICc) values and was, therefore, chosen as the best-fit substitution model for the construction of the tree. The neighbor-join (NJ) and BioNJ algorithms were applied to generate the initial tree for the heuristic ML search, and the partial deletion option was used for handling alignment gaps. Bootstrap analysis with 1000 replications was performed for assessing tree confidence.

Prediction of the Hex1 secondary structure was performed using the PSIPRED 4.0 Workbench (UCL-CS Bioinformatics, London, UK) [43]. For homology modeling of the Hex1 protein, the Hidden Markov Model-based tool HHPred [44] and MODELLER 9.25 [45] were used, based on the highly similar crystal structure of Hex1 from N. crassa (Protein Data Bank, PDB 1KHI) (probability: 99.93\%, E-value: $1.9 \times 10^{-23}$ ). Protein structures were visualized and compared using PyMOL 2.4 (https://pymol.org/2).

Statistical significance of differences between compared datasets was assessed using the analysis of variance (ANOVA) test, following the evaluation of homogeneity of variance across samples ( $F$-test $p$ $\leq 0.05$ ). Datasets were then subjected to means separation using Tukey's honestly significant difference (HSD) test. 


\section{Results}

\subsection{Identification and Characterization of the VdHex1 Homolog}

To identify homologs of Hex1 in $V$. dahliae, the 176 amino-acid (aa) protein sequence of $N$. crassa (GenBank AAB61278) was used as query in a tBlastN search of the $V$. dahliae genome (strain Ls.17). This yielded a single highly similar sequence of 197 aa (similarity: $80.0 \%$, E-value: $5.0 \times 10^{-91}$ ), encoded by the 786 bp long gene VDAG_01749. Sequence alignment revealed that this protein was missing the N-terminal hexapeptide MGYYDD, which is, however, highly conserved across Hex1 homologs of various ascomycetes. Further investigation of the gene model VDAG_01749 suggested the presence of a putative intron close to the start codon of the gene that could have been erroneously predicted and led to the description of a truncated ORF. The existence of this intron is conserved among various fungi [17-19]. To validate our hypothesis, we retrieved 120 reads from previously generated RNA-seq datasets by BLAST searches against the hex1 N-terminal region, and we mapped them on its genomic sequence (Figure 1A). More than half of them (67 reads) were mapped on both flanks of our predicted intron, with an internal gap of $217 \mathrm{bp}$, which corresponds to a genomic sequence with GT-AG boundaries. These findings are consistent with splicing of an intron at these positions, yielding a protein of 188 aa and molecular weight of $21 \mathrm{kDa}$. Twenty reads exhibited a different mapping pattern that suggests alternative splicing of the intron by using an internal AG donor site (Figure 1A) to produce a larger but highly similar isoform of the Hex1 protein (209 aa, $23.5 \mathrm{kDa}$ ). This has also been observed in a number of fungi $[18,21]$. Finally, 33 reads showed gapless alignments on the genomic sequence of hex1, suggesting the presence of pre-messenger RNAs (mRNAs) in the RNA-seq data. Both $V$. dahliae isoforms of Hex1 possess the typical N-terminal hexapeptide MGYYDD and the characteristic peroxisome-targeting signal (SRL tripeptide; PTS1 [16]) at their C-terminus (Figure S1, Supplementary Materials).
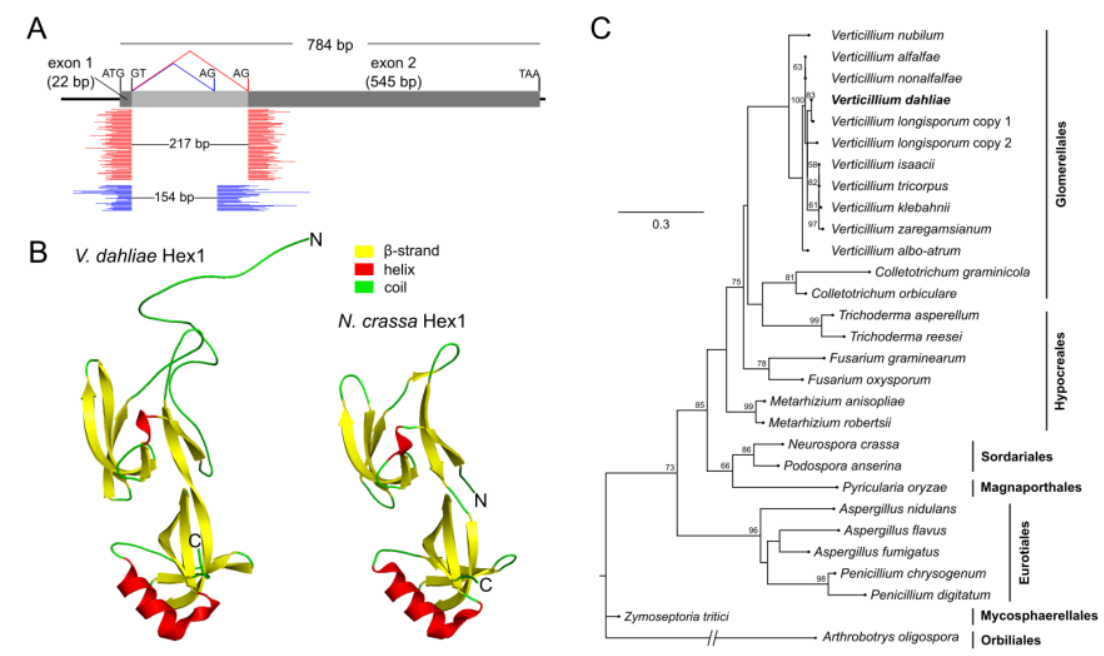

Figure 1. Gene and protein structure of the Hex1 homolog of Verticillium dahliae, and phylogenetic analysis. (A) Gene structure of locus VDAG_01749. Mapping of RNA-seq reads on the genomic sequence supports the definition of an intron that can be spliced in two alternative ways (red and blue reads, respectively) to produce two highly similar isoforms of the protein. (B) Prediction by homology modeling of the tertiary structure of $V$. dahliae Hex1 and comparison to the solved structure of its Neurospora crassa homolog (PDB 1KHI). (C) Maximum likelihood phylogenetic tree of Hex1 homologs across several Pezizomycotina orders of varying phylogenetic distances from Verticillium.

Multiple sequence alignment with Hex1 homologs of selected representative species revealed that, apart from the initial hexapeptide, the N-terminal region is highly variable across fungi (Figure S1, Supplementary Materials). In V. dahliae, as well as some other species, this region of the protein lacks the polyhistidine motif that has been shown to be involved in Hex1 targeting to the septal wall in Aspergillus 
fumigatus [13]. This suggests that different species have presumably adopted different mechanisms for recruiting WBs to the septum. The remaining part of the protein up to its C-terminus (which constitutes about $78 \%$ of the total protein length) is highly conserved, even between species of different orders. Secondary structure prediction revealed that $V$. dahliae Hex1 contains all characteristic motifs, including the 11 beta-sheets and two helices, as well as the conserved histidine residue (at position 51) which has been implicated in crystal formation in N. crassa [16] (Figure S1, Supplementary Materials). Furthermore, homology modeling of $V$. dahliae Hex 1 demonstrated that the protein is expected to have essentially the same tertiary structure as the solved structure of its N. crassa homolog (PDB $1 \mathrm{KHI}[16])$, notably with two perpendicular antiparallel $\beta$-barrels, except for the variable $\mathrm{N}$-terminal region, which is probably in a disordered state (Figure 1B).

Genomic searches revealed that all known Verticillium species possess a single Hex1 homolog, with the exception of $V$. longisporum, a nearly diploid hybrid [46], which has two nonidentical paralogs. Phylogenetic analysis of the Hex1 homologs of Verticillium and members of several Pezizomycotina orders of varying phylogenetic distances demonstrated that the evolution of this single-copy gene generally follows the divergence pattern of the species (Figure 1C).

\subsection{Deletion of Vdhex1 and Morphological and Physiological Characterization}

To determine the functions of Hex1 in V. dahliae, a deletion mutant ( $\Delta$ hex1) was constructed through double homologous recombination using Agrobacterium tumefaciens for transforming wild-type conidia (isolate Ls.17). Replacement of the wild-type hex1 gene with the neo cassette (conferring resistance to geneticin) was confirmed using PCR and Southern blot analyses (Figure S2, Supplementary Materials). The full-length hex 1 ORF of the wild-type isolate, flanked by $2 \mathrm{~kb}$ long genomic sequences, was amplified and co-transformed with plasmid pUCATPH to generate the complemented strain hex1-c.

When compared to the wild type and the complemented strain, the $\Delta$ hex 1 mutant exhibited significantly slower growth ( $p$-value: $\left.9.0 \times 10^{-4}\right)$, more compact aerial mycelium, and abnormal colony periphery, both on PDA and on MM (Figure 2A,B). Microsclerotia and pigment deposition in the knockout strain were delayed by 5 days; however, after 30 days of growth, they were indistinguishable from the other strains. The mutant exhibited significant phenotypic alterations regarding its asexual reproductive capacity. Conidiation was dramatically lower than in the wild type (10-fold reduction, $p$-value: $8.7 \times 10^{-7}$; Figure $2 \mathrm{~B}$ ), and the ability of its conidia to germinate was also significantly reduced ( $15.3 \%$ reduction, $p$-value: $1.8 \times 10^{-5}$ ) in comparison to the wild type (Figure $2 \mathrm{~B}$ ). Furthermore, we frequently observed abnormal morphologies in $\Delta$ hex 1 germlings, which included bipolar or irregular patterns of germ tube emergence, and faster branching of young germlings (Figure 2C). Mature $\Delta$ hex 1 hyphae and conidiophores also exhibited a distinctive "curly" phenotype in their apical regions, which often appeared wavy and fragile (Figure 2D; Figure S3, Supplementary Materials). All aforementioned phenotypes were fully rescued by complementation of hex 1 in the hex1-c strain, confirming their ascription to the hex 1 deletion. On the other hand, the mutant's septation pattern was not significantly altered (Figure 2D). 
A
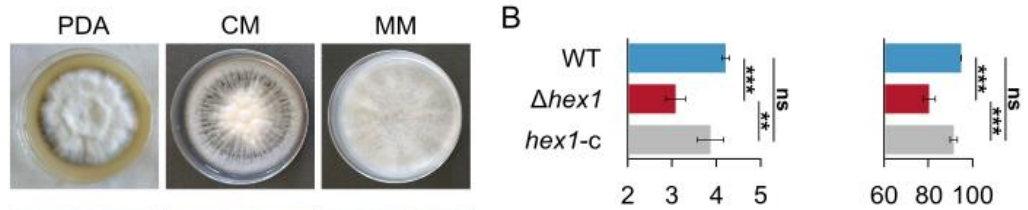

6080100

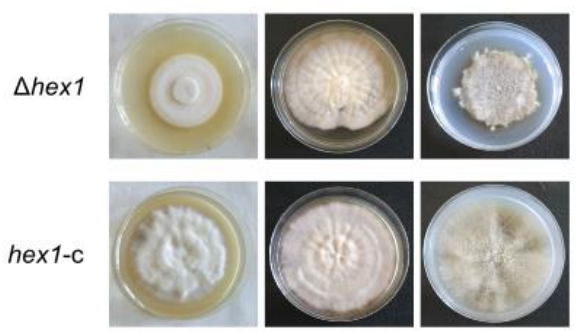

growth rate $(\mathrm{mm} / \mathrm{d}) \quad \%$ germination

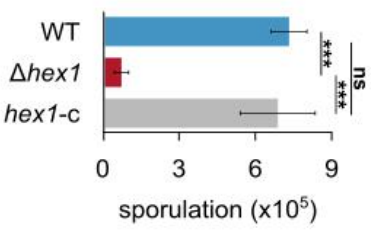

C

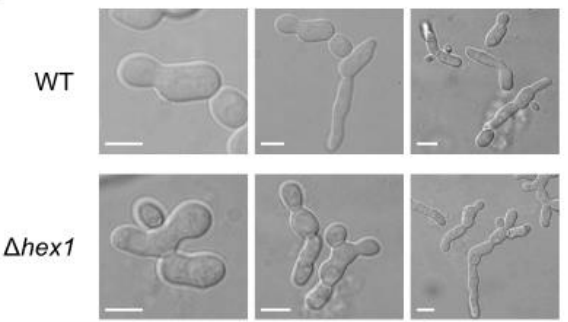

D
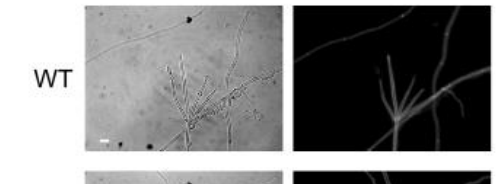

E

WT

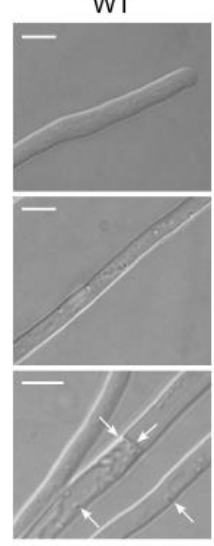

$\Delta h e x 1$

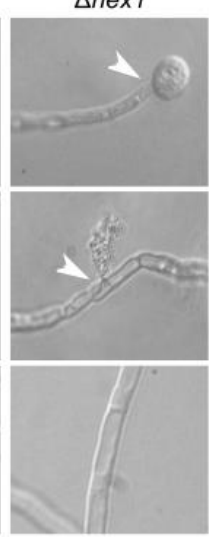

F
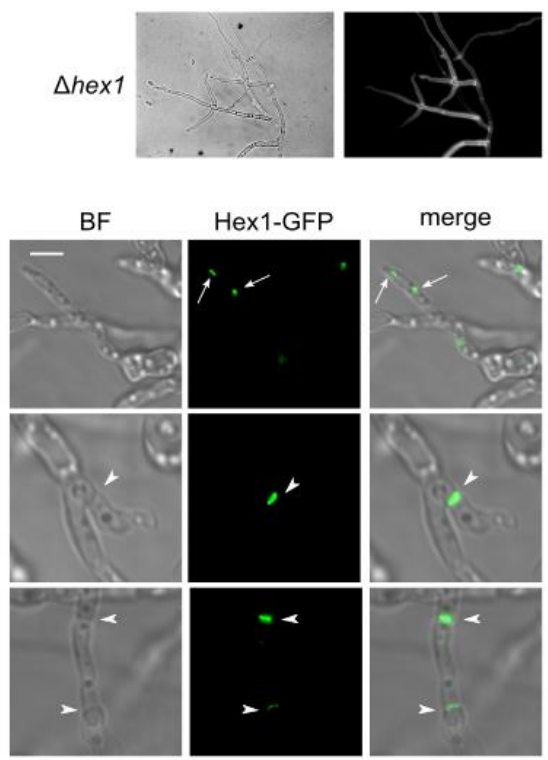

$\Delta h e x 1$

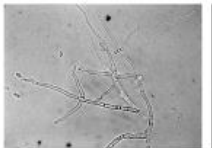

merge

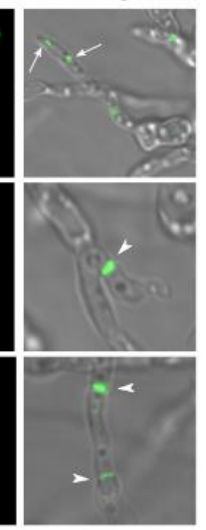

Figure 2. Morphological and physiological characterization of the $V$. dahliae $\Delta$ hex1 strain. (A) Colony morphologies of the $V$. dahliae wild-type isolate (Ls.17), the deletion mutant $\Delta$ hex1, and the complemented strain hex1-c, after growth for 25 days on PDA, CzD-CM, and MM. CM: CzD-CM. (B) Growth rate, ability of conidia to germinate, and abundance of conidial production of the three strains. All experiments were performed in triplicate, and 150 conidia were tested for germination per replicate. Bars: SD. Statistical significance of differences was tested by one-way ANOVA, followed by Tukey's post hoc test (** $p \leq 0.01,{ }^{* * *} p \leq 0.001$, ns: nonsignificant). (C,D) Microscopic characteristics of conidia (C; bars $\left.=5 \mu \mathrm{m}\right)$ and hyphae (D; cell wall staining using calcofluor white M2R; bar $=10 \mu \mathrm{m}$ ) of the wild-type and the $\Delta$ hex1 strains. (E) Response of the wild-type and the $\Delta$ hex 1 strains to hypotonic shock by immersing their hyphae to distilled water. Arrowheads: hyphal burst and cytoplasmic leakage. Arrows: spherical vesicles, usually localized close to the septal wall, that were observed during live-cell imaging of the wild-type strain but were absent from $\Delta$ hex1. Bars $=10 \mu \mathrm{m}$. (F) Subcellular localization of the Hex1-sGFP tagged protein in $V$. dahliae. Hex1 is localized either in small globular vesicles (presumably peroxisomes or Woronin bodies (WBs), indicated by arrows) or at the septal wall (arrowheads). Bar $=5 \mu \mathrm{m}$.

The three strains were subjected to hypotonic shock by immersing their hyphae into distilled water, in order to study their integrity and resilience. Notably, hyphal burst and cytoplasmic leakage were frequently observed only in the $\Delta$ hex 1 strain, mostly at the hyphal tips and rarely at subapical compartments, 
leading sometimes to shrunk hyphae that had lost their cytoplasm (Figure 2E). During live-cell imaging of hyphae of both the wild-type and the rescued strains, we also often observed spherical vesicles in the vicinity of septae (and rarely close to the cell wall without obvious connection to septae), which were not detected in $\Delta$ hex1. Overall, these results are consistent with the hypothesis that Hex1 in V. dahliae is essential for WB formation, which is involved in the maintenance of hyphal integrity, presumably by conditionally sealing the septal pore.

\subsection{Localization of VdHex1 at Septae}

Our finding that the $V$. dahliae Hex1 homolog lacks a polyhistidine motif in its N-terminal region, which has been demonstrated to be involved in septal targeting in A. fumigatus [13], motivated us to examine directly its subcellular localization. For this, we constructed a V. dahliae strain expressing a Hex1-sGFP construct under the control of its endogenous promoter. Fusion of sGFP at the C-terminus of Hex1 was expected to largely inactivate the terminal peroxisome signal motif [47]. Indeed, vesicles with sGFP signal, presumably corresponding to peroxisomes or WBs, were only relatively rarely observed (Figure 2F). On the other hand, strong fluorescence was often detected at septae; notably, this was not limited to septal pores, but was distributed along the entire septum (Figure 2F). These results directly demonstrate the association of Hex1 with septae in $V$. dahliae and suggest that different species have probably adopted different mechanisms for targeting Hex1 to the septum.

\subsection{Vdhex1 Is Indispensable for Pathogenicity}

Pathogenicity bioassays on eggplant (cv. Black Beauty), which is highly susceptible to V. dahliae [38], were performed to investigate the possible involvement of Hex1 in pathogenicity and virulence of the fungus. Eggplant seedlings were inoculated with the wild-type, $\Delta h e x 1$, and hex1-c strains, and their disease severity was assessed 45 days after inoculation. The $\Delta$ hex 1 mutant caused notably less severe disease compared to the wild-type and hex1-c strains (Figure 3A,B). Only a small percentage of $\Delta$ hex 1 -inoculated plants $(6.7 \%)$ showed severe symptoms (disease severity $>50 \%$ ), while the majority $(86.7 \%)$ remained symptom-free or exhibited very mild symptoms (disease severity $\leq 20 \%$ ). This was in contrast to the wild type and the complemented strains, which caused severe symptoms to $36.7 \%$ and $30.0 \%$ of plants, respectively, characterized by typical Verticillium-induced symptoms including wilting, chlorosis, and defoliation (Figure 3B).

To gain a better understanding of the pathogenic properties of the $\Delta$ hex 1 deletion mutant, we performed an additional detailed time-course comparison of virulence using pathogenicity assays of the wild type and $\Delta$ hex 1 strains over 29 days post inoculation (Figure 3C). Plants inoculated with the wild type started presenting symptoms on day 15 , and the mean disease severity at the end of the experiment was $59.4 \%( \pm 4.7 \%)$. On the contrary, infection with the $\Delta$ hex 1 mutant started causing symptoms only on day 22 , reaching a final severity of $9.1 \%( \pm 3.3 \%)$. Calculation of the area under disease progress curve (AUDPC) values, which account for the plant disease progression over 29 days, revealed that the $\Delta$ hex 1 virulence was 16.3 times lower than that of the wild type (Figure 3D). Consistently, the average fresh weight of plants treated with $\Delta$ hex1 was significantly higher than that of plants treated with the wild type (Figure 3E). Finally, we investigated the presence of the fungus within plant tissues by attempting to re-isolate it from xylem sections of the infected plants, at the end of the experiment. The re-isolation ratio of $\Delta$ hex 1 was reduced by $49.4 \%$ in comparison to the wild type $(0.41 \pm 0.09$ and $0.81 \pm 0.08$, respectively; Figure $3 \mathrm{~F})$, which corroborates the difference observed in disease severity and demonstrates the difficulty of the $\Delta$ hex 1 mutant to colonize the xylem and cause systemic infection of the plants. 
A

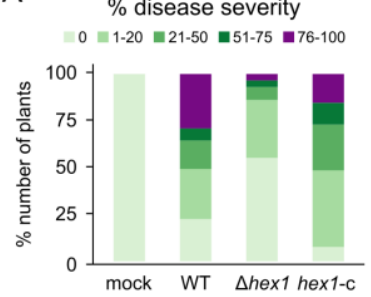

C

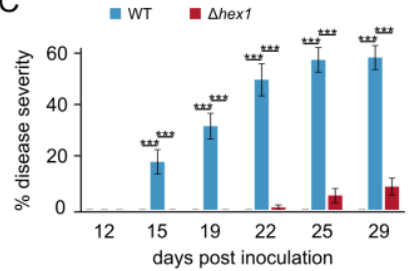

B

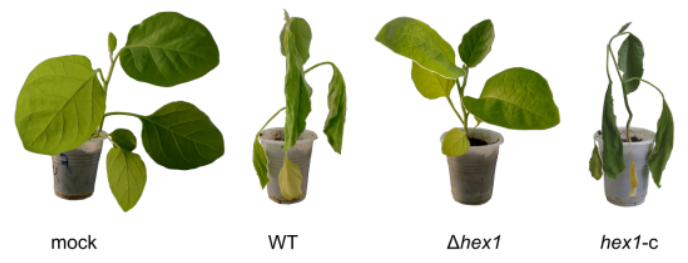

D

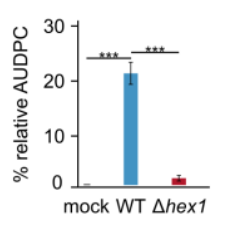

$\mathrm{E}$

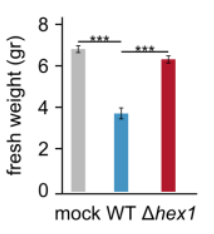

$\mathrm{F}$

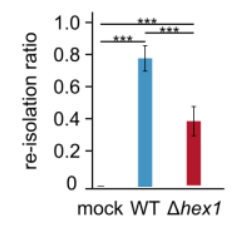

Figure 3. Phytopathological characterization of the V. dahliae $\Delta$ hex1 strain. (A) Disease severity of the wild-type (WT), the deletion ( $\Delta$ hex1), and the complemented (hex1-c) strains, 45 days post inoculation on eggplant seedlings (30 plants/strain). Uninfected plants (mock) served as control. (B) Representative plants are shown for each strain. (C) Time-course comparison of disease severity between the wild type and $\Delta$ hex1 (21 plants/strain) over 29 days post inoculation. (D-F) Mean relative area under disease progress curve (AUDPC) scores, plant fresh weights, and fungal re-isolation ratios at the end of the time-course experiment. Bars = standard error (SE). Statistical testing by one-way ANOVA followed by Tukey's post hoc test $(* * * 00.001)$.

\subsection{VdHex1 Is Involved in the Fungal Response to Osmotic Stress and Cell Wall-/Plasma} Membrane-Perturbating Agents

On the basis of our previous findings and the demonstrated functions of Hex1 and WBs in other fungi [27], we supposed that the Hex1 homolog of $V$. dahliae might also be involved in environmental stress responses and the maintenance of cellular integrity. To test this, we exposed the deletion mutant $\Delta$ hex 1 to hyperosmotic stress and a variety of cell wall-perturbating or damaging substances, and we compared its responses to those of the wild-type and the complemented strains by spot dilution assays and radial growth analyses. Upon treatment with either $\mathrm{NaCl}$ or sorbitol, the $\Delta$ hex 1 strain achieved remarkably reduced growth in comparison to the other strains, even though germination as revealed by our spot assays was not significantly affected (Figure 4A,B). The observed growth inhibition appeared dependent on the substance used (i.e., exposure to $0.5 \mathrm{M} \mathrm{NaCl}$ caused the same level of inhibition as 1.0 M sorbitol, Figure 4B), while our preliminary tests demonstrated that the extent of inhibition was also concentration-dependent. Microscopic observation of the three strains after growth in a hyperosmotic medium of $0.5 \mathrm{M} \mathrm{NaCl}$ revealed that many $\Delta$ hex 1 germlings exhibited hyphal bleeding of their cytoplasm, and the majority of them were entirely permeable to methylene blue, which stains dead cells. On the contrary, wild-type and rescued cells remained mostly unstained, or only one compartment allowed accumulation of the dye, while the remaining hypha was unaffected (Figure 4C). This suggests that Hex1 is involved in hyphal compartmentalization and ensures cell heterogeneity, presumably by occluding septal pores upon osmotic stress to protect the remaining hypha from destruction. Furthermore, hyperosmotic conditions induced increased septation in $\Delta$ hex1, as well as increased the frequency of "curly" hyphae with branching and the appearance of "bubble"-like cells, only in the mutant (Figure $4 \mathrm{C}$ ). These findings are further suggesting increased sensitivity of $\Delta$ hex 1 cells to osmotic stress. 
A

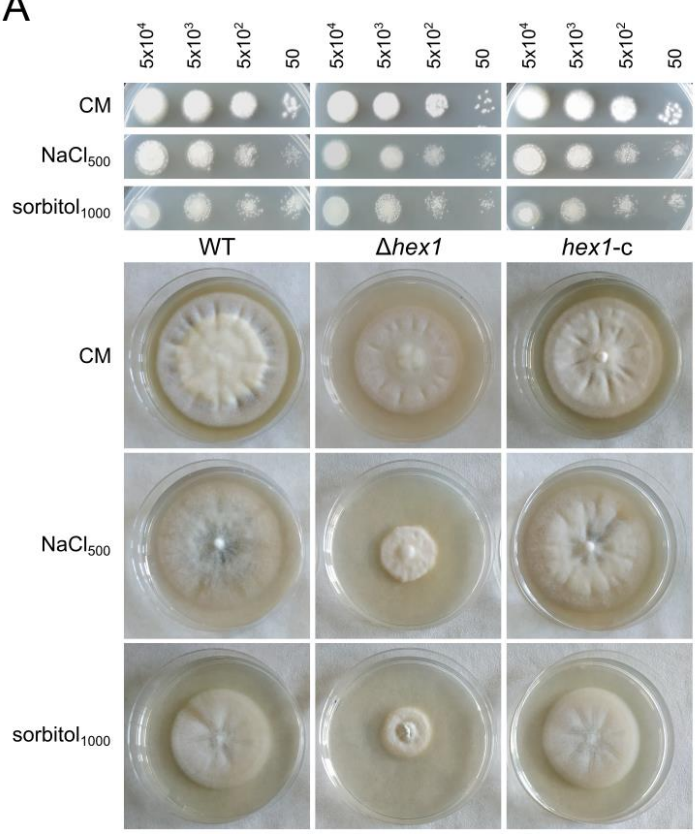

B

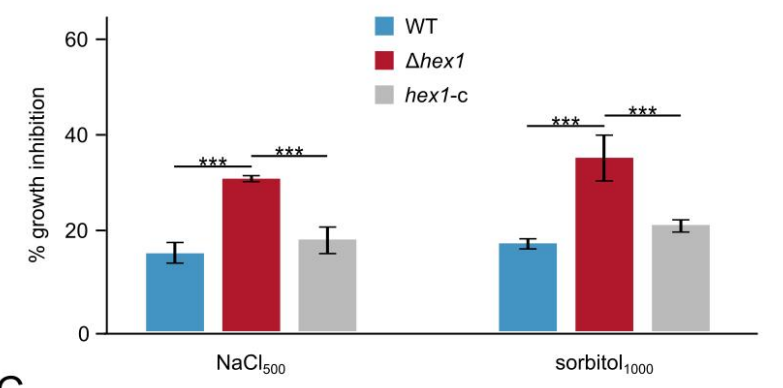

C
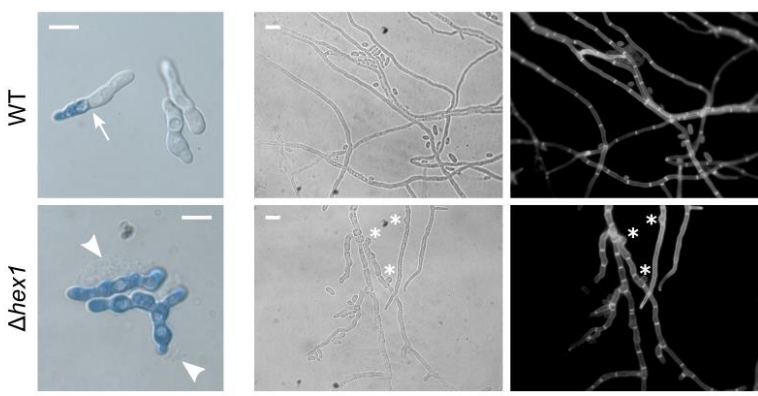

Figure 4. Effects of hyperosmotic stress on $\Delta$ hex1. (A) Responses of the wild-type, the deletion ( $\Delta$ hex1), and the complemented (hex1-c) strains to $\mathrm{NaCl}$ and sorbitol, with regard to germination (top; the number of inoculated conidia per spot are provided above the images; growth for 3 days) and radial growth (bottom; growth for 18 days). CM: CzD-CM. (B) Relative growth inhibition by $\mathrm{NaCl}$ and sorbitol (each condition was tested in triplicate; bars = SD; statistical testing by one-way ANOVA followed by Tukey's post hoc test $\left.{ }^{* * *} p \leq 0.001\right)$. All concentrations are given in $\mathrm{mM}$. (C) Staining of wild-type and $\Delta$ hex 1 germlings with methylene blue, after growth for $16 \mathrm{~h}$ in a hyperosmotic medium of $0.5 \mathrm{M}$ $\mathrm{NaCl}$ (left). Arrow: septum. Arrowheads: cytoplasmic bleeding. Morphology of corresponding hyphae under the same conditions. Asterisks: "bubble"-like cells. Cell wall staining using calcofluor white M2R. Bar $=10 \mu \mathrm{m}$.

Calcofluor white, Congo red, and SDS were used to test the sensitivity of the $\Delta$ hex1 mutant to cell wall- and membrane-damaging agents. Germination frequency of the mutant was slightly reduced in the presence of Congo red, and its radial growth was significantly limited upon exposure to calcofluor white and Congo red, suggesting reduced tolerance against cell wall stress (Figure 5A,B). It should be noted that the sensitivity of the $\Delta$ hex 1 mutant was remarkably higher to calcofluor white in comparison to Congo red (Figure 5A,B). Similar effects were observed when we tested the antifungal agents amphotericin $B$ and the acyclic sesquiterpene alcohol farnesol, which target sterol biosynthesis and membrane integrity (Figure 5A,B). Fluconazole was also tested but seemed to affect equally the deletion mutant and the control strains (Figure S4, Supplementary Materials).

Finally, we tested a number of substances that are present in commercially available fungicides as inhibitors of fungal sporulation and germination (cymoxanil, fosetyl-AI, mandipropamid, and cyflufenamid). All of them affected similarly the mutant and the control strains (Figure S4, Supplementary Materials). 

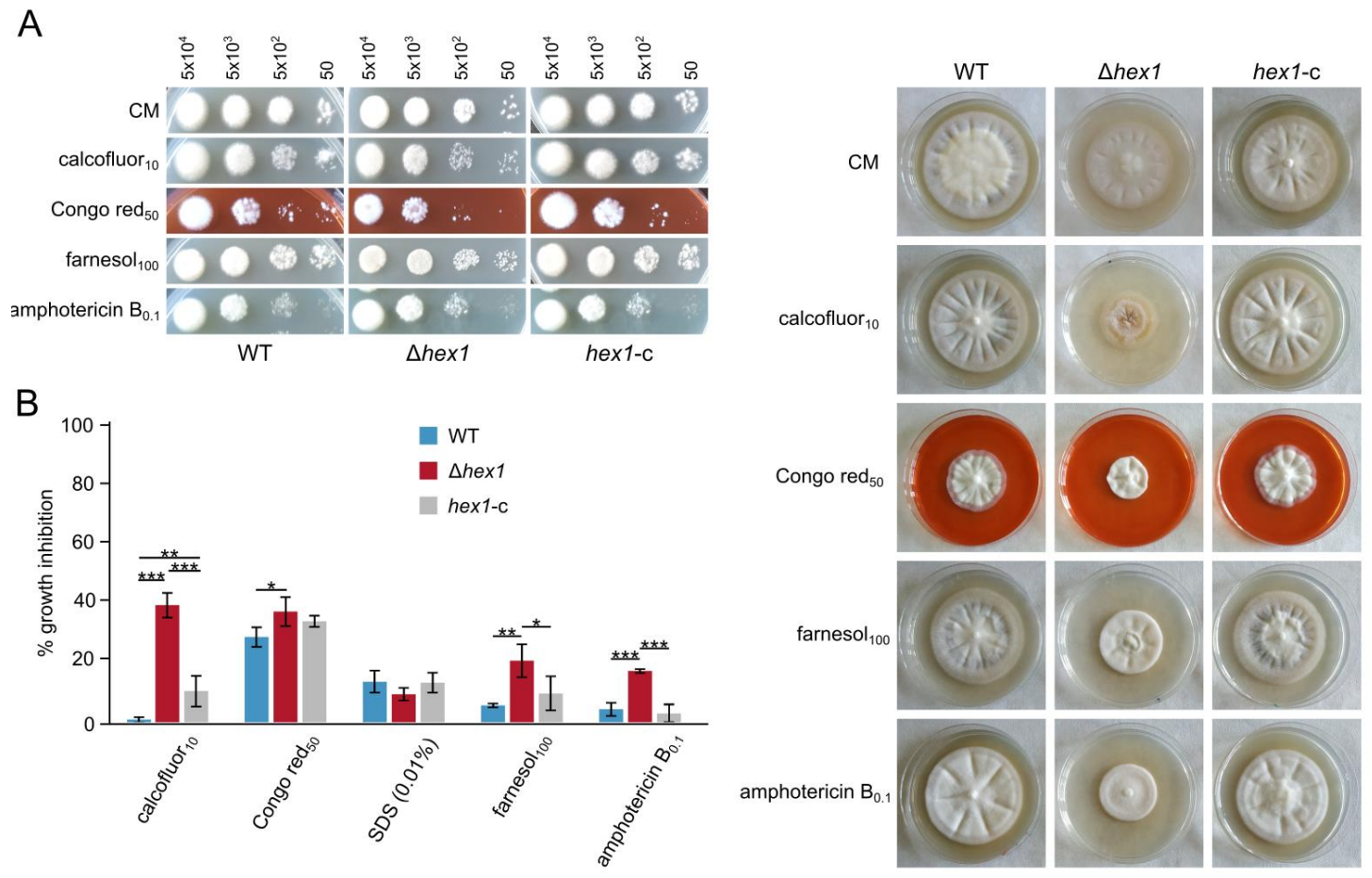

\section{B}

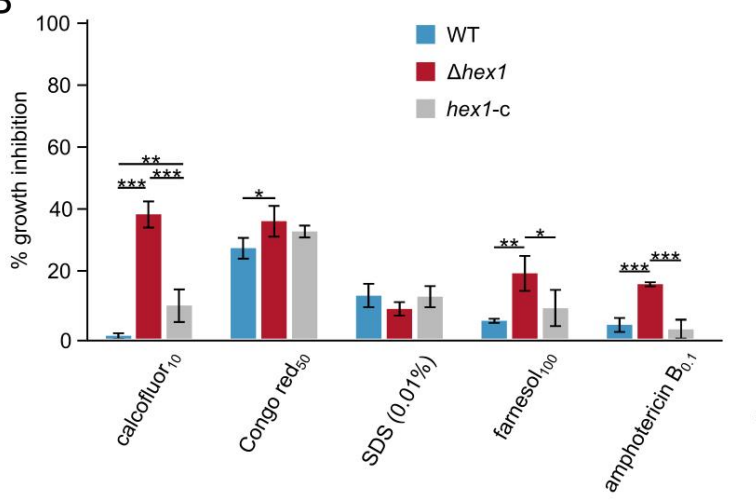

Figure 5. Effects of cell wall and plasma-membrane stress on $\Delta$ hex1. (A) Responses of the wild-type, the deletion ( $\Delta$ hex1), and the complemented (hex1-c) strains to calcofluor white M2R, Congo red, SDS, farnesol, and amphotericin B with regard to germination (top; the number of inoculated conidia per spot are provided above the images; growth for 3 days) and radial growth (right; growth for 18 days). CM: CzD-CM. (B) Relative growth inhibition by the same substances (each condition was tested in triplicate; bars $=\mathrm{SD}$; statistical testing by one-way ANOVA followed by Tukey's post hoc test ${ }^{*} p \leq 0.05$, ${ }^{* *} p \leq 0.01,{ }^{* * *} p \leq 0.001$ ). All concentrations are given in $\mu \mathrm{g} / \mathrm{mL}$.

\subsection{VdHex1 Is Required for Normal Response to Oxidative Stress and ROS Metabolism}

Understanding of the strategies that organisms have evolved to cope with oxidative stress, on one hand, and to use reactive oxygen species (ROS) as important signaling components in developmental regulation, on the other, are of fundamental interest; this becomes especially relevant in the case of pathogens, which need efficient mechanisms to counteract host-induced oxidative responses [48,49]. Since the role of Hex 1 in response to oxidative stress is not well established, we used the oxidative agents $\mathrm{H}_{2} \mathrm{O}_{2}$, the herbicide paraquat, and iprodione (i.e., a broad-spectrum antifungal drug that blocks germination and causes oxidative damage), as well as $\mathrm{N}$-acetyl cysteine as ROS scavenger, to examine the possible involvement of the protein in the resistance of the fungus to ROS. Treatment with any of the three ROS-inducing substances resulted in growth inhibition of the $\Delta$ hex 1 mutant and induced pigmentation only in the wild-type and complemented strains, while iprodione additionally caused a drastic reduction in germination frequency, particularly in $\Delta$ hex1 (Figure 6A,B). We note that, in the case of $\mathrm{H}_{2} \mathrm{O}_{2}$, significant differences between the null mutant and the other strains were only observed in intermediate concentrations (Figure 6A,B), while the same trend but lacking statistical significance was observed when concentrations lower than $0.7 \mathrm{mM}$ and higher than $2.0 \mathrm{mM}$ were tested. 
A
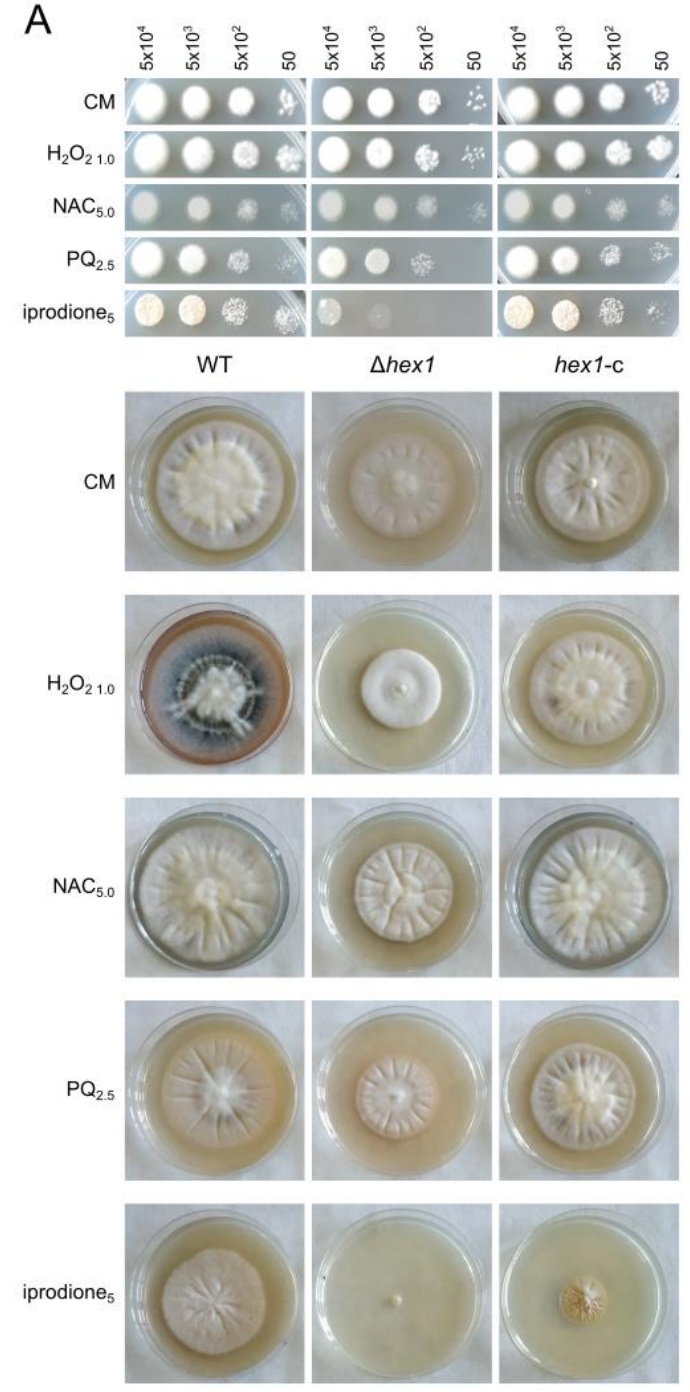

Figure 6. Effects of oxidative stress on $\Delta$ hex1 and its involvement in reactive oxygen species (ROS) metabolism. (A) Responses of the wild-type, the deletion ( $\Delta$ hex1), and the complemented (hex1-c) strains to $\mathrm{H}_{2} \mathrm{O}_{2}$, paraquat, iprodione, and $N$-acetyl cysteine with regard to germination (top; the number of inoculated conidia per spot are provided above the images; growth for 3 days) and radial growth (bottom; growth for 18 days). CM: CzD-CM. (B) Relative growth inhibition by the same substances (each condition was tested in triplicate; bars = SD; statistical testing by one-way ANOVA followed by Tukey's post hoc test $\left.{ }^{*} p \leq 0.05,{ }^{* *} p \leq 0.01,{ }^{* * *} p \leq 0.001\right)$. All concentrations are given in $\mathrm{mM}$, except for iprodione $(\mu \mathrm{g} / \mathrm{mL})$. (C) Detection of superoxide anion radicals $\left(\mathrm{O}_{2}{ }^{-}\right)$using nitro blue tetrazolium chloride (NBT) staining in colonies of the two strains upon treatment with various sources of stress ( $\mathrm{NaCl}$, sorbitol, paraquat, and farnesol). Both top and bottom views of plates are shown for every treatment.

To further investigate how Hex1 possibly affects ROS levels under different types and levels of stress, we used a histochemical method of NBT staining to detect superoxide radicals $\left(\mathrm{O}_{2}{ }^{-}\right)$in the mycelium. We indeed observed a different behavior regarding ROS accumulation between $\Delta$ hex 1 and the control strains. Under standard conditions (CM), as well as upon treatment with the stressors paraquat and farnesol, the mutant displayed more limited ROS levels, restricted to a narrow peripheral mycelial area of active hyphal growth (Figure 6C). In sharp contrast, exposure to hyperosmotic stress ( $\mathrm{NaCl}$ or sorbitol) resulted in drastically elevated ROS levels throughout the colony. These findings suggest involvement of Hex1 in the regulation of ROS metabolism as a function of environmental stress. 


\subsection{VdHex1 Is Not Involved in Heterokaryon Incompatibility}

Vegetative fusion of hyphae from different individuals to the formation of heterokaryons is strictly regulated by heterokaryon incompatibility systems. If the two fusion partners have genetic differences in their het loci, the heterokaryotic cell compartment is isolated from the rest of the mycelium and often destroyed through a targeted cell death reaction [7]. On the basis of our findings, which suggest that Hex 1 and WBs of $V$. dahliae are involved in hyphal compartmentalization, presumably by conditionally sealing septal pores upon mechanical damage or environmental stress, we hypothesized that they might also be responsible for the isolation of heterokaryotic cells when incompatibility reactions are triggered. To investigate this possibility, we first checked whether hex1 in $V$. dahliae is required for hyphal fusion or involved in the control or activation of the heterokaryon incompatibility reaction per se. For this, we used the $\Delta$ hex1 deletion mutant in compatibility assays with standard tester strains from all known compatibility groups of $V$. dahliae (VCGs). In these tests, complementary nitrate non-utilizing (nit) mutants of tested isolates are used in pairings on medium which would support only heterokaryotic growth. Therefore, nit 1 and nitM mutants of the $\Delta$ hex 1 strain of isolate Ls.17 were generated and used in assays with complementary nit mutants of 23 wild-type isolates of all compatibility groups; self-pairings (i.e., with independent nit mutants of the same wild-type isolate, Ls.17) were also performed as controls (Table S4, Supplementary Materials). The results are summarized in Table S4 (Supplementary Materials). Mutants of the $\Delta$ hex 1 strain were compatible with each other and gave rise to robust heterokaryons, suggesting that hex 1 is not required for hyphal fusion. Mutants of both the wild-type isolate Ls.17 and its $\Delta$ hex 1 descendant exhibited strong reactions with members of the same compatibility group, as expected, as well as weaker or inconsistent interactions with members of other groups, which is often observed in this species [40]. No difference in the compatibility profile of the $\Delta h e x 1$ mutant was recorded, suggesting that the gene is not involved in the genetic control of heterokaryon incompatibility.

We then addressed our hypothesis that Hex1 might be involved in the containment of the incompatibility-induced catastrophic reaction to the heterokaryotic cell, using time-lapse live-cell imaging of fusion events between incompatible strains. We chose the wild-type isolate BB, which is incompatible with Ls.17, and we also deleted its hex1 gene, using the same strategy as for our reference strain (Figure S2, Supplementary Materials). In addition, we labeled the nuclei of BB by tagging its histone H1 with sGFP and the cytoplasm of Ls.17 with cytoplasmically localized sGFP. This would permit the direct microscopic identification of the two strains in pairings and detection of fusion events between the two. Using this strategy and the two engineered strains, we detected eight independent fusion events between the two incompatible strains and recorded time-lapse movies $(24 \mathrm{~h})$ to study the cell fate upon fusion. In all cases, fusion was followed by gradual nuclear degradation and cell shrinkage, which are typical manifestations of heterokaryon incompatibility-induced cell death [7] (Figure 7A). These processes were always confined to the heterokaryotic cell, and they never affected the adjacent cell compartments (Figure 7A; Video S1, Supplementary Materials). These results suggest that WBs and their major component, Hex1, are not necessary for septal pore plugging in response to the activation of heterokaryon incompatibility cell death reactions. On the other hand, in all of these cases, staining with calcofluor white indicated highly increased chitin deposition on the septae which surround the heterokaryotic cell that is destroyed (Figure 7A,B; Figure S5 and Video S2, Supplementary Materials), as well as on the cell wall of the compartment (Figure S5, Supplementary Materials). Although it is not clear whether this has a role in the reaction or is just a consequence, a reasonable hypothesis is that this extreme thickening of septae might be ensuring the efficient sealing of the heterokaryotic cell and preventing the diffusion of cell death mediators to the adjacent cells. 
A

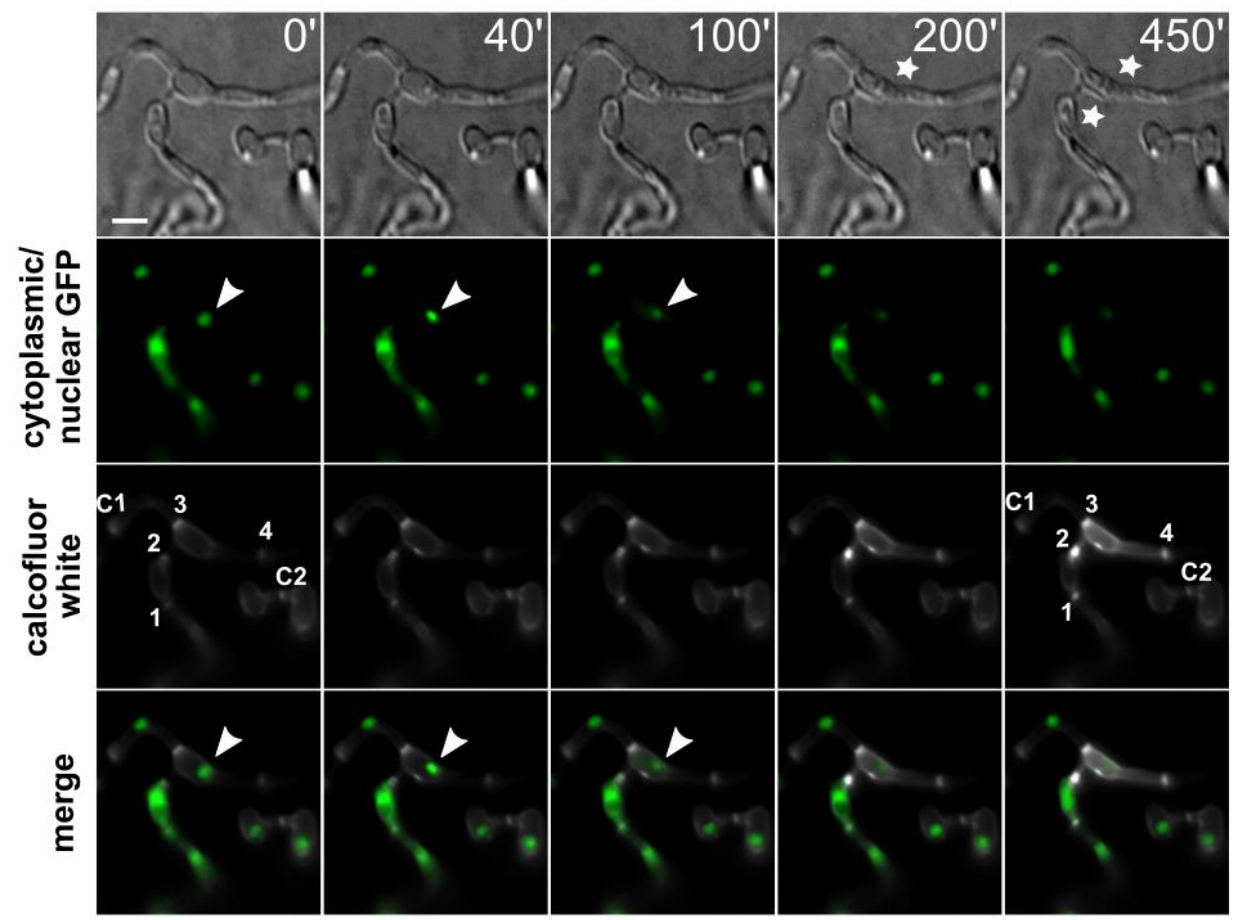

B
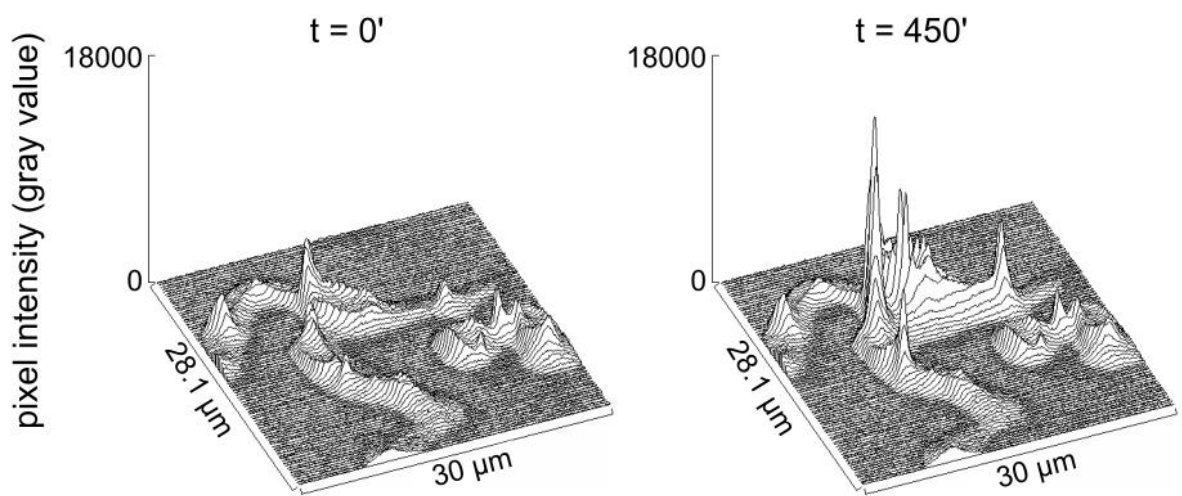

Figure 7. Vdhex 1 is not involved in heterokaryon incompatibility. (A) Time-lapse microscopic examination of a fusion event between the incompatible strains BB $\Delta$ hex1 H1-sGFP and Ls.17 sGFP. Typical characteristics of incompatibility-induced cell death, namely, nuclear degradation (arrowheads) and cell shrinkage (asterisks), are observed. Septae that surround the fused cells are indicated by numbers 1-4, and two control septae in neighboring hyphal compartments are indicated by C1-C2 (see also Figure S5, Supplementary Materials). Cell wall staining was performed using calcofluor white M2R. Bar $=10 \mu \mathrm{m}$. (B) Quantification of blue fluorescence intensity (calcofluor white M2R staining of cell wall chitin) in the region of interest shown in panel A, for two post-fusion time points, before and after the visible effects of the incompatibility reaction.

Finally, according to the established functions of WBs in controlling traffic through the septal pore of fungi, we contemplated that they could also be involved in the control of nuclear migration, an essential step to the formation of heterokaryons through hyphal fusions. However, microscopic examination of the BB $\triangle$ hex 1 strain with sGFP-labeled nuclei revealed that essentially all hyphal compartments were strictly uninucleate (Figure S6, Supplementary Materials), with the exception of the actively dividing cells at hyphal tips, which can transiently have more than one nuclei until the formation of the new septum. This was also the case in the wild-type strain. We rarely observed nuclei migrating through the septal pore, both in wild-type and in $\Delta$ hex1 hyphae (Video S3, Supplementary Materials). A possible 
explanation for the fact that binucleate cells are, nevertheless, never observed in the mycelium could involve degradation of one of the nuclei upon migration [50].

\section{Discussion}

Multicellularity has independently evolved from unicellular ancestors in different eukaryotic lineages, with diverse outcomes [51]. In filamentous fungi, these transitions led to the evolution of the syncytial mycelium, which is characterized by cytoplasmic continuity [52]. This ensures efficient translocation of nutrients and genetic resources [53] and coordination of responses to environmental changes [54], without the need for a dedicated vascular system. Nevertheless, uncontrolled intercompartmental traffic would lead to increased exposure to biotic and abiotic risks that could rapidly propagate throughout the mycelium [3-7], which underlines the necessity for a trade-off between continuity and conditional control of exchanges. Fungal evolution has invented structures and mechanisms that address this need, including septation of hyphae in higher fungi (ascomycetes and basidiomycetes) [2] and regulated mechanisms for the control of cytoplasmic flow through septal pores [8]. In ascomycetes, the peroxisome-derived WBs are involved in this process of plugging septal pores as a response to mechanical or other stimuli $[9,27,55]$.

The major component of WBs was shown to be the conserved peroxisomal protein Hex1 in N. crassa $[15,17]$. Its discovery was followed by detailed structural and functional analyses, mostly in this and to a lesser extent in other fungi $[13,16,18,20,26]$. In this study, we identified the single Hex1 homolog of $V$. dahliae, a plant pathogen with a particularly broad range of hosts and significant economic impact [28]. A careful study of the deposited gene model, using automatic annotation, led us to revise the exon-intron boundaries of the gene and further revealed that it is subject to alternative splicing, a process that appears to be very common in V. dahliae [56] and which, specifically for hex1, has also been reported in certain other fungi [17-19]. The expression of two protein isoforms has also been experimentally demonstrated in other species using Hex1-specific antibodies [17,18,21], which corroborates our suggestion. The inaccuracy of automatic gene annotation in this case might explain the predicted hypervariable $\mathrm{N}$-terminal extensions observed in many sequences from different species $[25,26]$, indicating that these data should be treated with caution.

Woronin bodies are peroxisomal vesicles with a dense core of self-assembled Hex1 into a crystal lattice [16]. Both $V$. dahliae isoforms of Hex1 were predicted to adopt essentially the same tertiary structure as their N. crassa homolog [16], with the exception of the N-terminal region, which is extremely variable in sequence between fungi and seems to remain in a disordered state. Typical peroxisomal targeting signal peptides [47] were detected at their C-termini, and the conserved histidine residue that is necessary for crystallization of the protein is also present [16]. None of the isoforms have a polyhistidine motif in the variable N-terminal region, which in A. fumigatus is necessary for septal targeting of Hex1 [13]. However, such motifs are also absent from other Hex1 homologs, including that of N. crassa [17]. Moreover, C-terminal tagging of the $V$. dahliae Hex1 with sGFP demonstrated that Hex1 is still targeted to the septum, despite the lack of this motif. Overall, these findings suggest that more than one mechanism mediates septal targeting of Hex 1 in fungi. On the basis of this localization pattern and the well-established role of Hex1 in septal pore plugging, we investigated the resilience of mutant $\Delta$ hex 1 hyphae. In full concordance with its expected function, the lack of Hex1 rendered $V$. dahliae hyphae prone to excessive hyphal bleeding upon hypotonic shock-induced hyphal damage. This suggests defects in hyphal compartmentalization due to dysfunctional septal pore sealing.

Growing evidence from various fungi indicates that, apart from its defining role in septal plugging upon damage, Hex1 has pleiotropic phenotypes in fungal morphology and physiology $[13,17,18,22,26]$. Some of these reports present contradictory results regarding particular phenotypes in different species, including growth characteristics and pathogenicity. These considerations served as our motivation to investigate a number of hypothesized roles of Hex1 in physiology, pathogenicity, stress response, ROS metabolism, and heterokaryon incompatibility in $V$. dahliae. Our results suggest the multifaceted involvement of Hex1 in several fundamental processes in this fungus. Deletion of hex1 led to reduced growth rate, similarly to N. crassa [15,17] and Arthrobotrys oligospora [24], unlike several 
other fungi, in which deletion of the gene did not result in growth defects $[18,21,22,25]$. The absence of Hex 1 from $V$. dahliae also resulted in drastic reduction in conidiogenesis, which has been generally observed $[17,22,24,25]$. Furthermore, we identified in $V$. dahliae developmental phenotypes that were hitherto unknown for this gene, namely, restricted and abnormal conidial germination, increased hyphal branching, "curly" hyphal tips, and "bubble"-like cells. Collectively, V. dahliae Hex1 appears to be involved in a number of fundamental processes that are related to asexual reproduction, hyphal development, and possibly cell wall deposition and cellular polarity. An enrichment of WBs in the apical region of germ tubes and hyphal tips has been described in N. crassa and A. nidulans [11,57,58], and their peroxisome-unassociated apical clustering has been shown in A. fumigatus [21]. These resemble the localization pattern of the Spitzenkörper, the organizing center of hyphal growth that coordinates the vesicle-mediated delivery of cell wall material to the apical cell surface [59]. These observations and the pleiotropic growth phenotypes that we report here open up the possibility that Hex1 and WBs might have multiple cellular roles in growth and morphogenesis of $V$. dahliae and other fungi, some of which might be independent from their established role in septal pore occlusion upon damage, a hypothesis that welcomes future investigation.

Motivated by the identification of phenotypes in $\Delta$ hex 1 cells that suggest reduced cell wall robustness, including the detection of hyphal bleeding from internal compartments upon hypotonic shock and the "curly" hyphal tips that were often observed, we further investigated the responses of the mutant strain to osmotic stress and to factors that affect cell wall and membrane integrity. We found that, apart from growth inhibition, presumably due to the reduced ability of the hyphae to isolate their damaged cellular compartments, hyperosmotic conditions also caused increased septation and frequent appearance of morphological alterations, further supporting the hypothesis that Hex1 contributes to the regulation of colony morphogenesis. Moreover, our experiments demonstrated that Hex 1 in $V$. dahliae mediates resistance against common fungicides that target the cell wall and the plasma membrane. In the absence of Hex1, we observed reduced germination of conidia and growth rate upon treatment with either calcofluor white or Congo red, which indicates that changes in cell wall structure and related defects in hyphal integrity possibly result in impaired germination. Contrasting results have been reported with regard to the sensitivity of $\Delta$ hex 1 mutant strains of other fungi to osmotic and cell wall/plasma-membrane stress; deletion of the gene did not significantly affect M. robertsii and A. flavus [25,26], whereas, in A. fumigatus and Arthrobotrys oligospora, it had a significant impact [13,24].

We further hypothesized and investigated whether Hex1 could be involved in the fungal response to oxidative stress and ROS metabolism. The mutant strain indeed exhibited growth defects in coping with increased oxidative damage induced by treatment with $\mathrm{H}_{2} \mathrm{O}_{2}$ and paraquat, as well as with amphotericin B, farnesol, and iprodione, but not fluconazole. This may indicate that its hyphal development might be affected not only by inhibition of ergosterol biosynthesis, but also by the oxidative damage that these factors can cause. Furthermore, NBT staining of superoxide in the mycelium indicated altered levels of ROS accumulation, both under standard conditions and in the presence of high oxidative load. Osmotic stress caused increased ROS accumulation in the $\Delta$ hex1 mycelia and this could possibly explain the significant developmental defects in these conditions. On the other hand, in the presence of farnesol, $\Delta$ hex 1 exhibited lower ROS generation. These findings open up the possibility that Hex1 plays a role in ROS metabolism and resistance to oxidative stress in V. dahliae. Considering the significance of intracellular ROS in signaling and regulatory functions [60], such a connection with Hex1 might be worth investigating further. Furthermore, it possibly becomes especially relevant in pathogenic fungi, which need to cope with host responses to infection that often involve bursts of ROS generation [48,49]. In the pathogenic fungi Alternaria brassicicola and A. fumigatus, WBs were shown to be associated with the redox homeostasis-related transmembrane protein TmpL [61], which could be consistent with a function of Hex1 in ROS metabolism. To our knowledge, we report here the first experimental data that suggest involvement of Hex1 in ROS homeostasis. 
Considering the pleiotropic and significant effects that Hex1 deletion has on fungal growth and development, one could reasonably further assume a possibly important role in pathogenicity. This has been investigated in several plant and other pathogens, and results between species are contradicting, demonstrating partial attenuation of virulence to varying degrees in some cases $[13,18,22,25]$, but no compromise in pathogenic capacity in others $[23,26]$. We report here, for $V$. dahliae, one of the most pronounced roles of Hex1 in fungal pathogenicity. Deletion of hex 1 diminished virulence of $V$. dahliae on eggplant, a very susceptible host of this pathogen [38]. Consistently with this drastically reduced disease severity (by $84.7 \%$ on average), presence of the fungus in the xylem of infected plants was also significantly reduced (by $49.4 \%$ ). These results suggest that Hex1 is required not only for penetration of roots by $V$. dahliae, but also for its propagation in the host and its ability to cause systemic infection. It is reasonable to assume that this drastic reduction of virulence could be linked to the pleiotropic defects of $\Delta h e x 1$ in $V$. dahliae growth, physiology, and stress response, rather than to solely attribute the attenuated virulence to an independent role of Hex1 in pathogenicity. The infection cycle of $V$. dahliae begins with germination of microsclerotia, elongation of hyphae, and penetration of roots. In this endeavor, hyphal integrity is indispensable for efficient attack to the plant [62]. We demonstrated that deletion of Hex1 negatively affected germination and hyphal growth, as well as cell wall integrity and resistance to stress conditions. Furthermore, propagation of the fungus in plant tissues, mostly within xylem vessels, is required in subsequent stages of the plant disease, which involves sequential conidiation, germination, and hyphal growth cycles [63]. However, we found that the Hex1-deficient mutant has a diminished ability to produce conidia, which would probably challenge its ability to efficiently colonize the infected plant [64]. Finally, it is possible that the lack of Hex1 and functional WBs might negatively interfere with the ability of the fungus to withstand the stressful adverse conditions induced in the xylem by the plant's defense mechanisms. For example, one of the host responses to fungal invasion involves rapid bursts of ROS [48]. Since $\Delta$ hex 1 cells are defective in their responses to oxidative stress, this could be an additional factor that compromises its pathogenic potential.

Establishment of viable heterokaryons in fungi, resulting from hyphal fusion between different individuals, is often prevented in natural populations by incompatibility mechanisms. One of them involves the triggering of a cell death reaction if the fused cells are genetically incompatible, resulting in intense vacuolization, nuclear degradation, cell wall shrinkage, and, finally, cell death [7,33]. However, this reaction is highly localized and only affects the fused cells, whereas the adjacent hyphal compartments remain unaffected [7]. We hypothesized that the mechanism that is responsible for sealing of the incompatible fused cells to prevent the diffusion of cell death mediators to neighboring cells might involve the WBs, in a function analogous to septal pore plugging upon mechanical damage. However, our time-lapse imaging experiments of incompatible fusions clearly demonstrated that this compartmentalization function is independent of Hex1, as it normally occurs in $\Delta$ hex 1 hyphae. This is similar to what was previously observed in N. crassa [65]. In the course of our experiments, we invariably observed the fusion-induced thickening of septae defining the boundaries of the fused incompatible cells, presumably by intense chitin deposition. This was also detected, to a lesser extent, in the cell wall of these cells, but never affected any of the adjacent hyphal compartments. Similar observations were previously made in Podospora anserina [66]. We propose that the reinforcement of the septae of incompatible cells is involved in their sealing mechanism, which aims at preventing the incompatibility reaction from spreading in the hyphae, as well as protecting the neighboring cells once their fused neighbor gets disorganized as the result of the incompatibility reaction.

In conclusion, we demonstrate here important and pleiotropic roles of the protein Hex 1 in the plant pathogenic fungus $V$. dahliae. Apart from its function in hyphal compartmentalization in response to hyphal damage, involvement of the protein was identified in fundamental biological processes related to fungal growth, physiology, asexual reproduction, stress response, and pathogenicity. Although Hex1 is highly conserved in ascomycetes, diverse functions have been attributed to its homologs in different species, which underlines the necessity of further functional investigations to fully elucidate its multifaceted role in fungal biology. 
Supplementary Materials: The following are available online at http://www.mdpi.com/2309-608X/6/4/344/s1: Figure S1. Multiple sequence alignment of Hex1 homologs of $V$. dahliae and other selected ascomycetes, for comparison; Figure S2. Schematic representation of the strategy followed for hex1 deletion in V. dahliae; Figure S3. Morphological characteristics of hyphae of the wild-type and the $\Delta$ hex1 strains; Figure S4. Effects of antifungal agents on $\Delta$ hex1; Figure S5. Vdhex1 is not involved in heterokaryon incompatibility; quantification of blue fluorescence intensity (calcofluor white M2R staining of cell wall chitin) of septae and cross-section of the fused cells (additional to Figure 7); Figure S6. Nuclear copy number per hyphal compartment in mycelia of the $V$. dahliae BB wild-type and $\Delta$ hex1 strains; Table S1. Verticillium dahliae strains constructed and used in this study; Table S2. List of DNA oligonucleotides used in this study; Table S3. List of plasmids constructed and used in this study; Table S4. Results from the heterokaryon compatibility tests between nit mutants of $V$. dahliae isolates; Video S1. Time-lapse microscopic examination of a fusion event between the incompatible strains BB Shex1 H1-sGFP and Ls.17 sGFP (additional to Figure 7A); Video S2. Time-lapse quantification of blue fluorescence intensity (calcofluor white M2R staining of cell wall chitin) in the region of interest shown in Video S1 (additional to Figure 7B); Video S3. Nuclear migration through the septal pore, in BB $\Delta$ hex1 H1-sGFP (calcofluor white M2R staining of cell wall chitin).

Author Contributions: Conceptualized the project, M.A.T. and I.A.P.; designed and performed the experiments, V.V., I.A.P., and E.A.M.; designed and performed the bioinformatic analyses, I.A.P. and V.V.; interpreted and discussed the results, V.V., I.A.P., and M.A.T.; prepared the draft manuscript, V.V. and I.A.P.; discussed and revised the manuscript for submission, I.A.P., M.A.T., V.V., and E.A.M.; provided resources, M.A.T. and M.K. All authors read and agreed to the published version of the manuscript.

Funding: This research was co-financed by Greece and the European Union (European Social Fund- ESF) through the Operational Program "Human Resources Development, Education, and Lifelong Learning" in the context of the project "Strengthening Human Resources Research Potential via Doctorate Research" (MIS-5000432), implemented by the State Scholarships Foundation (IK $\Upsilon$ ).

Acknowledgments: We are grateful to E.K. Ligoxigakis (NAGREF-DEMETER, Heraklion, Greece) for his valuable help with the pathogenicity bioassays. Plasmids pSD1, pOSCAR, and pMF357 were generous gifts from Q.B. Nguyen (Kobe University, Japan), S.J. Klosterman (USDA-ARS, Salinas, USA), and E.A. Souza (Federal University of Lavras, Brazil), respectively. Agrobacterium tumefaciens AGL-1 was kindly provided by K. Haralampidis (National and Kapodistrian University of Athens, Greece). Plasmids pGKO2 and pFC332 were obtained from Addgene.

Conflicts of Interest: The authors declare no conflict of interest. The funders had no role in the design of the study; in the collection, analyses, or interpretation of data; in the writing of the manuscript, or in the decision to publish the results.

\section{References}

1. Fiddy, C.; Trinci, A.P. Mitosis, septation, branching and the duplication cycle in Aspergillus nidulans. J. Gen. Microbiol. 1976, 97, 169-184. [CrossRef] [PubMed]

2. Stajich, J.E.; Berbee, M.L.; Blackwell, M.; Hibbett, D.S.; James, T.Y.; Spatafora, J.W.; Taylor, J.W. The Fungi. Curr. Biol. 2009, 19, R840-R845. [CrossRef] [PubMed]

3. Roberson, R.W.; Abril, M.; Blackwell, M.; Letcher, P.; McLaughlin, D.J.; Mouriño-Pérez, R.R.; Riquelme, M.; Uchida, M. Hyphal structure. In Cellular and Molecular Biology of Filamentous Fungi; Borkovich, K., Eboole, D., Eds.; ASM Press: Washington, DC, USA, 2010; pp. 8-27.

4. Bartnicki-Garcia, S. Hyphal tip growth outstanding questions. In Molecular Biology of Fungal Development; Osiewacz, H., Ed.; Marcel Dekker Inc.: New York, NY, USA, 2002; pp. 29-58.

5. Nuss, D.L. Hypovirulence: Mycoviruses at the fungal-plant interface. Nat. Rev. Microbiol. 2005, 3, 632-642. [CrossRef] [PubMed]

6. Van Diepeningen, A.D.; Debets, A.J.M.; Slakhorst, S.M.; Hoekstra, R.F. Mitochondrial pAL2-1 plasmid homologs are senescence factors in Podospora anserina independent of intrinsic senescence. Biotechnol. J. 2008, 3, 791-802. [CrossRef]

7. Gonçalves, A.P.; Heller, J.; Daskalov, A.; Videira, A.; Glass, N.L. Regulated Forms of Cell Death in Fungi. Front. Microbiol. 2017, 8, 1837. [CrossRef]

8. Trinci, A.P.J.; Collinge, A.J. Structure and plugging of septa of wild type and spreading colonial mutants of Neurospora crassa. Archiv für Mikrobiologie 1973, 91, 355-364. [CrossRef]

9. Markham, P.; Collinge, A.J. Woronin bodies of filamentous fungi. FEMS Microbiol. Lett. 1987, 46, 1-11. [CrossRef] 
10. Bleichrodt, R.-J.; van Veluw, G.J.; Recter, B.; Maruyama, J.; Kitamoto, K.; Wösten, H.A.B. Hyphal heterogeneity in Aspergillus oryzae is the result of dynamic closure of septa by Woronin bodies: Hyphal heterogeneity is maintained by septal closure. Mol. Microbiol. 2012, 86, 1334-1344. [CrossRef]

11. Liu, F.; Ng, S.K.; Lu, Y.; Low, W.; Lai, J.; Jedd, G. Making two organelles from one: Woronin body biogenesis by peroxisomal protein sorting. J. Cell Biol. 2008, 180, 325-339. [CrossRef]

12. Ng, S.K.; Liu, F.; Lai, J.; Low, W.; Jedd, G. A Tether for Woronin Body Inheritance Is Associated with Evolutionary Variation in Organelle Positioning. PLoS Genet. 2009, 5, e1000521. [CrossRef]

13. Beck, J.; Echtenacher, B.; Ebel, F. Woronin bodies, their impact on stress resistance and virulence of the pathogenic mould Aspergillus fumigatus and their anchoring at the septal pore of filamentous Ascomycota. Mol. Microbiol. 2013, 89, 857-871. [CrossRef] [PubMed]

14. Lai, J.; Koh, C.H.; Tjota, M.; Pieuchot, L.; Raman, V.; Chandrababu, K.B.; Yang, D.; Wong, L.; Jedd, G. Intrinsically disordered proteins aggregate at fungal cell-to-cell channels and regulate intercellular connectivity. Proc. Natl. Acad. Sci. USA 2012, 109, 15781-15786. [CrossRef] [PubMed]

15. Jedd, G.; Chua, N.-H. A new self-assembled peroxisomal vesicle required for efficient resealing of the plasma membrane. Nat. Cell Biol. 2000, 2, 226-231. [CrossRef] [PubMed]

16. Yuan, P.; Jedd, G.; Kumaran, D.; Swaminathan, S.; Shio, H.; Hewitt, D.; Chua, N.-H.; Swaminathan, K. A HEX-1 crystal lattice required for Woronin body function in Neurospora crassa. Nat. Struct. Mol. Biol. 2003, 10, 264-270. [CrossRef]

17. Tenney, K.; Hunt, I.; Sweigard, J.; Pounder, J.I.; McClain, C.; Bowman, E.J.; Bowman, B.J. hex-1, a Gene Unique to Filamentous Fungi, Encodes the Major Protein of the Woronin Body and Functions as a Plug for Septal Pores. Fungal Genet. Biol. 2000, 31, 205-217. [CrossRef]

18. Soundararajan, S.; Jedd, G.; Li, X.; Ramos-Pamploña, M.; Chua, N.H.; Naqvi, N.I. Woronin Body Function in Magnaporthe grisea Is Essential for Efficient Pathogenesis and for Survival during Nitrogen Starvation Stress. Plant Cell 2004, 16, 1564-1574. [CrossRef]

19. Curach, N.C.; Te'o, V.S.J.; Gibbs, M.D.; Bergquist, P.L.; Nevalainen, K.M.H. Isolation, characterization and expression of the hex1 gene from Trichoderma reesei. Gene 2004, 331, 133-140. [CrossRef]

20. Maruyama, J.; Juvvadi, P.R.; Ishi, K.; Kitamoto, K. Three-dimensional image analysis of plugging at the septal pore by Woronin body during hypotonic shock inducing hyphal tip bursting in the filamentous fungus Aspergillus oryzae. Biochem. Biophys. Res. Commun. 2005, 331, 1081-1088. [CrossRef]

21. Beck, J.; Ebel, F. Characterization of the major Woronin body protein HexA of the human pathogenic mold Aspergillus fumigatus. Int. J. Med. Microbiol. 2013, 303, 90-97. [CrossRef]

22. Son, M.; Lee, K.-M.; Yu, J.; Kang, M.; Park, J.M.; Kwon, S.-J.; Kim, K.-H. The HEX1 Gene of Fusarium graminearum is Required for Fungal Asexual Reproduction and Pathogenesis and for Efficient Viral RNA Accumulation of Fusarium graminearum Virus 1. J. Virol. 2013, 87, 10356-10367. [CrossRef]

23. Kubo, Y.; Fujihara, N.; Harata, K.; Neumann, U.; Robin, G.P.; O'Connell, R. Colletotrichum orbiculare FAM1 Encodes a Novel Woronin Body-Associated Pex22 Peroxin Required for Appressorium-Mediated Plant Infection. mBio 2015, 6, e01305-15. [CrossRef] [PubMed]

24. Liang, L.; Gao, H.; Li, J.; Liu, L.; Liu, Z.; Zhang, K.-Q. The Woronin body in the nematophagous fungus Arthrobotrys oligospora is essential for trap formation and efficient pathogenesis. Fungal Biol. 2017, 121, 11-20. [CrossRef] [PubMed]

25. Yuan, J.; Li, D.; Qin, L.; Shen, J.; Guo, X.; Tumukunde, E.; Li, M.; Wang, S. HexA is required for growth, aflatoxin biosynthesis and virulence in Aspergillus flavus. BMC Mol. Biol. 2019, 20, 4. [CrossRef] [PubMed]

26. Tang, G.; Shang, Y.; Li, S.; Wang, C. MrHex1 is Required for Woronin Body Formation, Fungal Development and Virulence in Metarhizium robertsii. J. Fungi 2020, 6, 172. [CrossRef] [PubMed]

27. Maruyama, J.; Kitamoto, K. The Woronin Body: A Fungal Organelle Regulating Multicellularity. In Biology of the Fungal Cell; Hoffmeister, D., Gressler, M., Eds.; Springer International Publishing: Cham, Switzerland, 2019; Volume 8, pp. 3-14.

28. Pegg, G.F.; Brady, B.L. Verticillium Wilts; CABI Pub.: Wallingford, UK, 2002.

29. Klosterman, S.J.; Atallah, Z.K.; Vallad, G.E.; Subbarao, K.V. Diversity, Pathogenicity, and Management of Verticillium Species. Annu. Rev. Phytopathol. 2009, 47, 39-62. [CrossRef] [PubMed]

30. Typas, M.A.; Heale, J.B. Transfer of a Cytoplasmic Factor by Micro-injection in Verticillium. J. Gen. Microbiol. 1979, 111, 375-386. [CrossRef] 
31. Typas, M.A. Heterokaryon incompatibility and interspecific hybridization between Verticillium albo-atrum and Verticillium dahliae following protoplast fusion and microinjection. Microbiology 1983, 129, 3043-3056. [CrossRef]

32. Typas, M.A.; Heale, J.B. Heterozygous diploid analyses via the parasexual cycle and a cytoplasmic pattern of inheritance in Verticillium spp. Genet. Res. 1978, 31, 131-144. [CrossRef]

33. Paoletti, M. Vegetative incompatibility in fungi: From recognition to cell death, whatever does the trick. Fungal Biol. Rev. 2016, 30, 152-162. [CrossRef]

34. Papaioannou, I.A.; Ligoxigakis, E.K.; Vakalounakis, D.J.; Markakis, E.A.; Typas, M.A. Phytopathogenic, morphological, genetic and molecular characterization of a Verticillium dahliae population from Crete, Greece. Eur. J. Plant Pathol. 2013, 136, 577-596. [CrossRef]

35. Papaioannou, I.A.; Dimopoulou, C.D.; Typas, M.A. Structural and phylogenetic analysis of the rDNA intergenic spacer region of Verticillium dahliae. FEMS Microbiol. Lett. 2013, 347, 23-32. [CrossRef] [PubMed]

36. Holsters, M.; de Waele, D.; Depicker, A.; Messens, E.; van Montagu, M.; Schell, J. Transfection and transformation of Agrobacterium tumefaciens. Mol. Gen. Genet. 1978, 163, 181-187. [CrossRef] [PubMed]

37. Mullins, E.D.; Chen, X.; Romaine, P.; Raina, R.; Geiser, D.M.; Kang, S. Agrobacterium-Mediated Transformation of Fusarium oxysporum: An Efficient Tool for Insertional Mutagenesis and Gene Transfer. Phytopathology 2001, 91, 173-180. [CrossRef]

38. Markakis, E.A.; Ligoxigakis, E.K.; Avramidou, E.V.; Tzanidakis, N. Survival, Persistence, and Infection Efficiency of Verticillium dahliae Passed Through the Digestive System of Sheep. Plant Dis. 2014, 98, 1235-1240. [CrossRef] [PubMed]

39. Markakis, E.A.; Fountoulakis, M.S.; Daskalakis, G.C.; Kokkinis, M.; Ligoxigakis, E.K. The suppressive effect of compost amendments on Fusarium oxysporum f.sp. radicis-cucumerinum in cucumber and Verticillium dahliae in eggplant. Crop Prot. 2016, 79, 70-79. [CrossRef]

40. Papaioannou, I.A.; Typas, M.A. High-Throughput Assessment and Genetic Investigation of Vegetative Compatibility in Verticillium dahliae. J. Phytopathol. 2015, 163, 475-485. [CrossRef]

41. Schindelin, J.; Arganda-Carreras, I.; Frise, E.; Kaynig, V.; Longair, M.; Pietzsch, T.; Preibisch, S.; Rueden, C.; Saalfeld, S.; Schmid, B.; et al. Fiji: An open-source platform for biological-image analysis. Nat. Methods 2012, 9, 676-682. [CrossRef]

42. Kumar, S.; Stecher, G.; Li, M.; Knyaz, C.; Tamura, K. MEGA X: Molecular Evolutionary Genetics Analysis across Computing Platforms. Mol. Biol. Evol. 2018, 35, 1547-1549. [CrossRef]

43. Buchan, D.W.A.; Jones, D.T. The PSIPRED Protein Analysis Workbench: 20 years on. Nucleic Acids Res. 2019, 47, W402-W407. [CrossRef]

44. Zimmermann, L.; Stephens, A.; Nam, S.-Z.; Rau, D.; Kübler, J.; Lozajic, M.; Gabler, F.; Söding, J.; Lupas, A.N.; Alva, V. A Completely Reimplemented MPI Bioinformatics Toolkit with a New HHpred Server at its Core. J. Mol. Biol. 2018, 430, 2237-2243. [CrossRef]

45. Webb, B.; Sali, A. Comparative Protein Structure Modeling Using MODELLER. Curr. Protoc. Protein Sci. 2016, 86, 2.9.1-2.9.37. [CrossRef] [PubMed]

46. Inderbitzin, P.; Davis, R.M.; Bostock, R.M.; Subbarao, K.V. The Ascomycete Verticillium longisporum is a Hybrid and a Plant Pathogen with an Expanded Host Range. PLoS ONE 2011, 6, e18260. [CrossRef] [PubMed]

47. Gould, S.J.; Keller, G.A.; Hosken, N.; Wilkinson, J.; Subramani, S. A conserved tripeptide sorts proteins to peroxisomes. J. Cell Biol. 1989, 108, 1657-1664. [CrossRef] [PubMed]

48. Lamb, C.; Dixon, R.A. The Oxidative Burst in Plant Disease Resistance. Annu. Rev. Plant Physiol. Plant Mol. Biol. 1997, 48, 251-275. [CrossRef] [PubMed]

49. Di Pietro, A.; Talbot, N.J. Fungal pathogenesis: Combatting the oxidative burst. Nat. Microbiol. 2017, $2,17095$. [CrossRef]

50. Corral-Ramos, C.; Roca, M.G.; Di Pietro, A.; Roncero, M.I.G.; Ruiz-Roldán, C. Autophagy contributes to regulation of nuclear dynamics during vegetative growth and hyphal fusion in Fusarium oxysporum. Autophagy 2015, 11, 131-144. [CrossRef]

51. Knoll, A.H. The Multiple Origins of Complex Multicellularity. Annu. Rev. Earth Planet. Sci. 2011, 39, $217-239$. [CrossRef]

52. Carlie, M.J.; Watkinson, S.C. The Fungi, 2nd ed.; Academic Press: London, UK, 2001.

53. Simonin, A.; Palma-Guerrero, J.; Fricker, M.; Glass, N.L. Physiological Significance of Network Organization in Fungi. Eukaryot. Cell 2012, 11, 1345-1352. [CrossRef] 
54. Schmieder, S.S.; Stanley, C.E.; Rzepiela, A.; van Swaay, D.; Sabotič, J.; Nørrelykke, S.F.; deMello, A.J.; Aebi, M.; Künzler, M. Bidirectional Propagation of Signals and Nutrients in Fungal Networks via Specialized Hyphae. Curr. Biol. 2019, 29, 217-228.e4. [CrossRef]

55. Steinberg, G.; Harmer, N.J.; Schuster, M.; Kilaru, S. Woronin body-based sealing of septal pores. Fungal Genet. Biol. 2017, 109, 53-55. [CrossRef]

56. Jin, L.; Li, G.; Yu, D.; Huang, W.; Cheng, C.; Liao, S.; Wu, Q.; Zhang, Y. Transcriptome analysis reveals the complexity of alternative splicing regulation in the fungus Verticillium dahliae. BMC Genom. 2017, 18, 130. [CrossRef] [PubMed]

57. Tey, W.K.; North, A.J.; Reyes, J.L.; Lu, Y.F.; Jedd, G. Polarized Gene Expression Determines Woronin Body Formation at the Leading Edge of the Fungal Colony. Mol. Biol. Cell 2005, 16, 2651-2659. [CrossRef] [PubMed]

58. Momany, M.; Richardson, E.A.; Van Sickle, C.; Jedd, G. Mapping Woronin Body Position in Aspergillus nidulans. Mycologia 2002, 94, 260. [CrossRef] [PubMed]

59. Riquelme, M.; Bredeweg, E.L.; Callejas-Negrete, O.; Roberson, R.W.; Ludwig, S.; Beltrán-Aguilar, A.; Seiler, S.; Novick, P.; Freitag, M. The Neurospora crassa exocyst complex tethers Spitzenkörper vesicles to the apical plasma membrane during polarized growth. Mol. Biol. Cell 2014, 25, 1312-1326. [CrossRef]

60. Heller, J.; Tudzynski, P. Reactive oxygen species in phytopathogenic fungi: Signaling, development, and disease. Annu. Rev. Phytopathol. 2011, 49, 369-390. [CrossRef]

61. Kim, K.-H.; Willger, S.D.; Park, S.-W.; Puttikamonkul, S.; Grahl, N.; Cho, Y.; Mukhopadhyay, B.; Cramer, R.A.; Lawrence, C.B. TmpL, a Transmembrane Protein Required for Intracellular Redox Homeostasis and Virulence in a Plant and an Animal Fungal Pathogen. PLoS Pathog. 2009, 5, e1000653. [CrossRef]

62. Qi, X.; Li, X.; Guo, H.; Guo, N.; Cheng, H. VdPLP, A Patatin-Like Phospholipase in Verticillium dahliae, Is Involved in Cell Wall Integrity and Required for Pathogenicity. Genes 2018, 9, 162. [CrossRef]

63. Fradin, E.F.; Thomma, B.P.H.J. Physiology and molecular aspects of Verticillium wilt diseases caused by V. dahliae and V. albo-atrum. Mol. Plant Pathol. 2006, 7, 71-86. [CrossRef]

64. Luo, X.; Mao, H.; Wei, Y.; Cai, J.; Xie, C.; Sui, A.; Yang, X.; Dong, J. The fungal-specific transcription factor Vdpf influences conidia production, melanized microsclerotia formation and pathogenicity in Verticillium dahliae. Mol. Plant Pathol. 2016, 17, 1364-1381. [CrossRef]

65. Hutchison, E.; Brown, S.; Tian, C.; Glass, N.L. Transcriptional profiling and functional analysis of heterokaryon incompatibility in Neurospora crassa reveals that reactive oxygen species, but not metacaspases, are associated with programmed cell death. Microbiology 2009, 155, 3957-3970. [CrossRef]

66. Dementhon, K.; Paoletti, M.; Pinan-Lucarré, B.; Loubradou-Bourges, N.; Sabourin, M.; Saupe, S.J.; Clavé, C. Rapamycin Mimics the Incompatibility Reaction in the Fungus Podospora anserina. Eukaryot. Cell 2003, 2, 238-246. [CrossRef] [PubMed]

Publisher's Note: MDPI stays neutral with regard to jurisdictional claims in published maps and institutional affiliations.

(C) 2020 by the authors. Licensee MDPI, Basel, Switzerland. This article is an open access article distributed under the terms and conditions of the Creative Commons Attribution (CC BY) license (http://creativecommons.org/licenses/by/4.0/). 